\title{
Comparative transcriptome and histological analyses of wheat in response to phytotoxic aphid Schizaphis graminum and non-phytotoxic aphid Sitobion avenae feeding
}

\author{
Yong Zhang ${ }^{1}$, Yu Fu' ${ }^{1}$, Jia Fan ${ }^{1}$, Qian $\mathrm{Li}^{1}$, Frédéric Francis ${ }^{2}$ and Julian Chen ${ }^{1 *}$
}

\begin{abstract}
Background: Infestation of the phytotoxic aphid Schizaphis graminum can rapidly induce leaf chlorosis in susceptible plants, but this effect is not observed with the nonphytotoxic aphid Sitobion avenae. However, few studies have attempted to identify the different defence responses induced in wheat by S. graminum and S. avenae feeding and the mechanisms underlying the activation of chlorosis by S. graminum feeding.

Results: S. graminum feeding significantly reduced the chlorophyll content of wheat leaves, and these effects were not observed with S. avenae. A transcriptomic analysis showed that the expression levels of genes involved in the salicylic acid, jasmonic acid and ethylene signalling defence pathways were significantly upregulated by both S. avenae and S. graminum feeding; however, more plant defence genes were activated by S. graminum feeding than S. avenae feeding. The transcript levels of genes encoding cell wall-modifying proteins were significantly increased after $S$. graminum feeding, but only a few of these genes were induced by S. avenae. Furthermore, various reactive oxygen species-scavenging genes, such as 66 peroxidase (POD) and 8 ascorbate peroxidase ( $A P x)$ genes, were significantly upregulated after S. graminum feeding, whereas only $15 P O D$ and one APx genes were induced by S. avenae feeding. The activity of four antioxidant enzymes was also significantly upregulated by $S$. graminum feeding. Cytological examination showed that $\mathrm{S}$. graminum feeding induced substantial hydrogen peroxide $\left(\mathrm{H}_{2} \mathrm{O}_{2}\right)$ accumulation in wheat leaves. The chlorosis symptoms and the loss of chlorophyll observed in wheat leaves after $\mathrm{S}$. graminum feeding were reduced and inhibited by the scavenging of $\mathrm{H}_{2} \mathrm{O}_{2}$ by dimethylthiourea, which indicated that $\mathrm{H}_{2} \mathrm{O}_{2}$ plays important role in the induction of chlorosis by S. graminum feeding.

Conclusions: S. graminum and S. avenae feeding induces the JA, SA and ET signalling pathways, but S. graminum activated stronger plant defence responses than S. avenae. S. graminum feeding triggers strong ROS-scavenging activity and massive $\mathrm{H}_{2} \mathrm{O}_{2}$ production in wheat leaves, and the accumulation of $\mathrm{H}_{2} \mathrm{O}_{2}$ induced by $\mathrm{S}$. graminum feeding is involved in the activation of chlorosis in wheat leaves. These results enhance our understanding of mechanisms underlying aphid-wheat interactions and provide clues for the development of aphid-resistant wheat varieties.
\end{abstract}

Keywords: Schizaphis graminum, Sitobion avenae, Wheat, Transcriptome, Cytological, Defense responses, Reactive oxygen species scavengers, Hydrogen peroxide, Chlorosis

\footnotetext{
* Correspondence: chenjulian@caas.cn

${ }^{1}$ State Key Laboratory for Biology of Plant Diseases and Insect Pests, Institute

of Plant Protection, Chinese Academy of Agricultural Sciences, Beijing

100193, People's Republic of China

Full list of author information is available at the end of the article
}

(c) The Author(s). 2019 Open Access This article is distributed under the terms of the Creative Commons Attribution 4.0 International License (http://creativecommons.org/licenses/by/4.0/), which permits unrestricted use, distribution, and reproduction in any medium, provided you give appropriate credit to the original author(s) and the source, provide a link to the Creative Commons license, and indicate if changes were made. The Creative Commons Public Domain Dedication waiver (http://creativecommons.org/publicdomain/zero/1.0/) applies to the data made available in this article, unless otherwise stated. 


\section{Background}

Through interactions with insects over a hundred million years, plants have evolved complex and accurate defence mechanisms against herbivores. In response to herbivory, plants can perceive damage-associated molecular patterns (DAMPs) or herbivory-associated molecular patterns (HAMPs) in insect oral secretions and subsequently induce direct and indirect plant defence responses [1,2]. Direct defences involve the production and accumulation of plant defensive chemicals, such as plant secondary metabolites (PSMs), proteinase inhibitors, polyphenol oxidases and other defensive proteins, which are induced by herbivory and reduce herbivore performance $[3,4]$. Indirect defences include the synthesis and release of complex blends of volatiles that attract parasitoids and predators of the herbivores $[5,6]$.

Insects from different feeding guilds tend to elicit distinct plant defensive strategies of plants [7, 8]. For example, leaf-eating beetles (Coleoptera) or caterpillars (Lepidoptera) cause extensive tissue damage during herbivory, which usually activates the jasmonic acid (JA)mediated defence pathway in plants [9, 10]. Different from leaf chewing insects, hemipterans, such as aphids and whiteflies, have highly modified piecing-sucking mouthparts (stylets) that can penetrate the extracellular pathway and feed on the nutrients from phloem sap provided by sieve elements (SEs). Although the stylets puncture through most parts of plant cells during the probing track, these insects cause less damage to cells than leaf-chewing herbivores [11]. Many studies have demonstrated that hemipteran feeding results in the induction of the salicylic acid (SA)-dependent defence pathway in plants $[12,13]$.

The grain aphid Sitobion avenae and the greenbug Schizaphis graminum (Hemiptera: Aphididae) are considered two important pests of wheat and other cereals worldwide, as they suck phloem sap and serve as vectors of the barley yellow dwarf virus (BYDV), resulting in significant yield losses [14, 15]. Aphids are classified as nonphytotoxic or phytotoxic according to the symptoms and damage caused by their feeding [16]. Similar to most aphid species, S. avenae are nonphytotoxic, and no typical symptoms of plant damage are rapidly induced by their feeding processes. However, infestation by $S$. graminum, a phytotoxic species, can immediately induce obvious leaf chlorosis in susceptible plants, resulting in the deterioration of plant quality and even in plant death $[17,18]$. Several studies have characterized the plant defence responses induced by infestation of $S$. avenae and S. graminum. For example, Zhao et al. found that S. avenae feeding induces the expression of several genes involved in both the SA- and JA-mediated defence pathways in wheat [19], and Zhu-Salzman et al. demonstrated that S. graminum feeding strongly induces the expression of genes involved in the SA-dependent pathway in sorghum (Sorghum bicolor) but weakly increases the expression levels of JA-related defence genes, such as lipoxidase $(L O X)$ and proteinase inhibitors (PIs) [20]. Using a cDNA microarray, several transcripts with significantly different expression patterns were identified in sorghum seedlings after S. graminum feeding, and these herbivore-responsive genes were mainly associated with photosynthesis, the biosynthesis of defence molecules, cell wall fortification, oxidative bursts and stress [21].

Previous studies have also shown that S. graminum feeding significantly increases the concentrations of amino acids, particularly essential amino acids, in the phloem of wheat and barley, and the enhancement of the nutritional quality of plants induced by S. graminum feeding improves the aphid performance [22]. It has been proposed that leaf-senescence-like changes triggered by $S$. graminum feeding are associated with the nutritional enhancement of host plants [18, 23]. Feeding damage caused by phytotoxic aphids is usually observed in susceptible hosts; therefore, it has been hypothesized that the nutritional enhancement of plants derived from senescence-like feeding damage is a strategy used by phytotoxic aphids to potentially counteract the negative effects of induced plant resistance, which would eventually improve aphid fitness on the host [18].

However, few studies have attempted to identify the molecular basis of the necrosis symptoms in wheat triggered by the phytotoxic aphid $S$. graminum and the different defence responses induced by $S$. graminum and $S$. avenae feeding. In this study, we integrated gene expression profiling through high-throughput RNA sequencing (RNA-Seq) with cytological examination to reveal the responses of wheat leaves to $S$. graminum and $S$. avenae feeding, to compare the differences in the metabolic pathways affected by the two cereal aphids and to uncover the mechanism underlying the induction of damage symptoms by $S$. graminum.

\section{Results}

Damage symptoms and changes in the chlorophyll content of wheat leaves after S. avenae and S. graminum feeding

As shown in Fig. 1a-c, no obvious damage symptoms were detected in leaves $48 \mathrm{~h}$ after $S$. avenae infestation compared with leaves without aphid infestation, whereas S. graminum feeding caused severe chlorosis in wheat leaves. Delayed fluorescence, which was used as a direct indicator of the chlorophyll content, was also measured in the wheat leaves $48 \mathrm{~h}$ after aphid infestation. As demonstrated in Fig. 1d-f, the untreated leaves and the $S$. avenae-infested leaves exhibited strong signals of delayed fluorescence, whereas low signals of delayed fluorescence were detected in the wheat leaves infested 

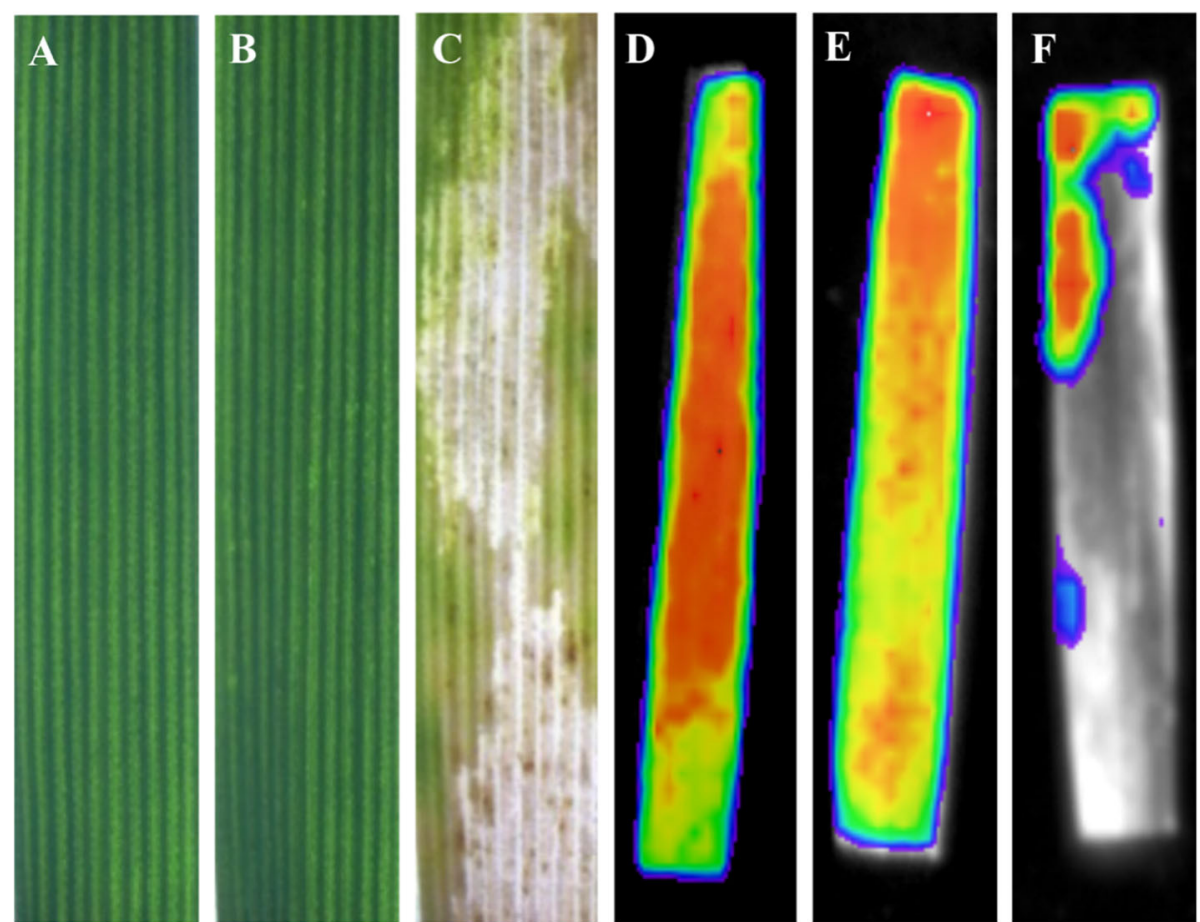

Fig. 1 Detection of damage symptoms (a-c) and delayed fluorescence (d-f) of wheat leaves after aphid infestation. Delayed fluorescence was detected using NightShade LB 985 In vivo Plant Imaging System. Experiments were performed with three biological replicates with similar results, and representative results from one replicate are shown. Red color indicates high intensities representing high chlorophyll content, blue color indicated low intensities of fluorescence, indicating low amounts of chlorophyll. No delayed fluorescence indicates destroyed chlorophyll. a and d: untreated leaves; $\mathbf{b}$ and $\mathbf{e}$ leaves infested with S. avenae for $48 \mathrm{~h}$; $\mathbf{c}$ and $\mathbf{f}$ : leaves infested with S. graminum for $48 \mathrm{~h}$

with S. graminum, which suggested the occurrence of chlorophyll degradation.

The chlorophyll content of the leaves after aphid feeding was further investigated. As shown in Fig. 2, no significant differences in the chlorophyll content were found between the $S$. avenae-infested and control plants.

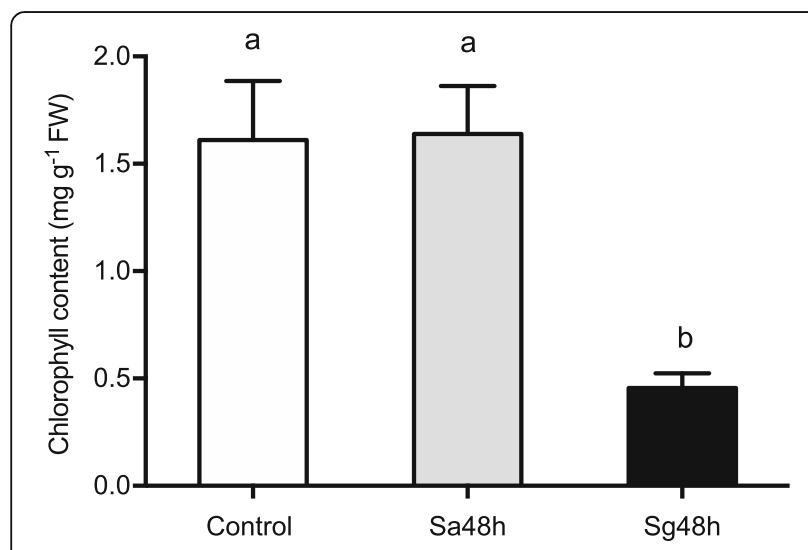

Fig. 2 Chlorophyll content of wheat leaves after aphid infestation. Control: untreated leaves; Sa48h: leaves infested with S. avenae for 48 h; Sg48h: leaves infested with S. graminum for $48 \mathrm{~h}$. The values are presented as means \pm SE of three biological replicates. Different letters indicate significant differences among treatments $(P<0.05$, ANOVA)
However, the chlorophyll content was significantly decreased to $0.46 \pm 0.068\left(\mathrm{~F}_{2,6}=10.494, P=0.011\right)$ after $S$. graminum feeding.

\section{Transcriptome data from aphid-infested wheat leaves}

The transcriptomes of wheat leaves infested with the two cereal aphid species were compared in this study. A total of $62.98 \mathrm{~Gb}$ of clean data were obtained from the nine leaf samples, and each of these samples contained $\geq 6.91 \mathrm{~Gb}$ with Q30 quality scores of $\geq 94.82 \%$ (Additional file 1: Table S1). Subsequently, 83.5 to $94.3 \%$ of the clean reads from each sample were aligned onto the wheat reference genome and matched to either unique or multiple genomic locations (Table 1).

\section{Identification and functional annotation of DEGs}

The gene expression levels of each replicate were assessed through principal component analysis (PCA). Replicates from the same group were clustered closely together, which suggested that the repeatability of each treatment group was satisfactory, and the samples from the S. avenae- and S. graminum-infested groups (Sa48h and $\mathrm{Sg} 48 \mathrm{~h}$, respectively) clustered far from the control samples, which indicated that aphid feeding induced significant changes in gene expression (Fig. 3). A total of 
Table 1 Mapping statistics of transcriptome database. The number in brackets indicates the percentage of total reads mapping to the wheat reference genome and/or matched at either multiple or unique genomic loci

\begin{tabular}{lllll}
\hline Sample name & Total reads & Total mapped & Multiple mapped & Uniquely mapped \\
\hline Control-1 & $46,272,278$ & $42,946,912(92.81 \%)$ & $4,147,714(8.96 \%)$ & $38,799,198(83.85 \%)$ \\
Control-2 & $46,669,950$ & $43,353,788(92.89 \%)$ & $4,598,942(9.85 \%)$ & $38,754,846(83.04 \%)$ \\
Control-3 & $47,129,428$ & $44,452,968(94.32 \%)$ & $4,238,840(8.99 \%)$ & $40,214,128(85.33 \%)$ \\
Sa48h-1 & $46,058,314$ & $43,100,126(93.58 \%)$ & $3,812,164(8.28 \%)$ & $39,287,962(85.3 \%)$ \\
Sa48h-2 & $46,068,740$ & $42,785,254(92.87 \%)$ & $3,721,176(8.08 \%)$ & $39,064,078(84.8 \%)$ \\
Sa48h-3 & $46,611,206$ & $43,536,070(93.4 \%)$ & $3,934,960(8.44 \%)$ & $39,601,110(84.96 \%)$ \\
Sg48h-1 & $46,754,444$ & $39,079,032(83.58 \%)$ & $3,388,782(7.25 \%)$ & $35,690,250(76.34 \%)$ \\
Sg48h-2 & $47,294,858$ & $43,947,242(92.92 \%)$ & $3,980,232(8.42 \%)$ & $39,967,010(84.51 \%)$ \\
Sg48h-3 & $47,136,410$ & $43,851,752(93.03 \%)$ & $3,760,518(7.98 \%)$ & $40,091,234(85.05 \%)$ \\
\hline
\end{tabular}

12,8195 transcripts were detected across all the samples (Additional file 2: Data S1). Gene expression levels with an adjusted $P$ value $<0.00001$ and $\mid \log _{2}$ Fold Change $\mid \geq$ 1 were selected as DEGs for further analysis. Forty-eight hours of $S$. avenae feeding significantly upregulated 1718 genes and significantly downregulated 172 genes in wheat leaves (Fig. 4a). In addition, 7893 and 5098 genes were significantly upregulated and downregulated, respectively, after $48 \mathrm{~h}$ of $S$. graminum feeding (Fig. $4 \mathrm{~b}$ ).

To investigate the differences in plant responses to infestation by $S$. graminum and $S$. avenae, the DEGs in wheat leaves induced by these aphids were also compared in our study. The results showed that the expression levels of 857 genes in wheat leaves were significantly upregulated by both $S$. graminum and $S$. avenae feeding. Additionally, 11,046 and 1914 transcripts were specifically and significantly upregulated after $S$. graminum and $S$. avenae feeding for $48 \mathrm{~h}$, respectively (Fig. 5a). In contrast, a total of 128 transcripts were significantly downregulated after both S. graminum and $S$. avenae infestation, and 7036 and 861 genes were only significantly downregulated after $S$. graminum and $S$. avenae feeding, respectively (Fig. $5 \mathrm{~b}$ ). This finding suggested that the global response of wheat to S. graminum feeding is distinct from that of wheat to $S$. avenae feeding.

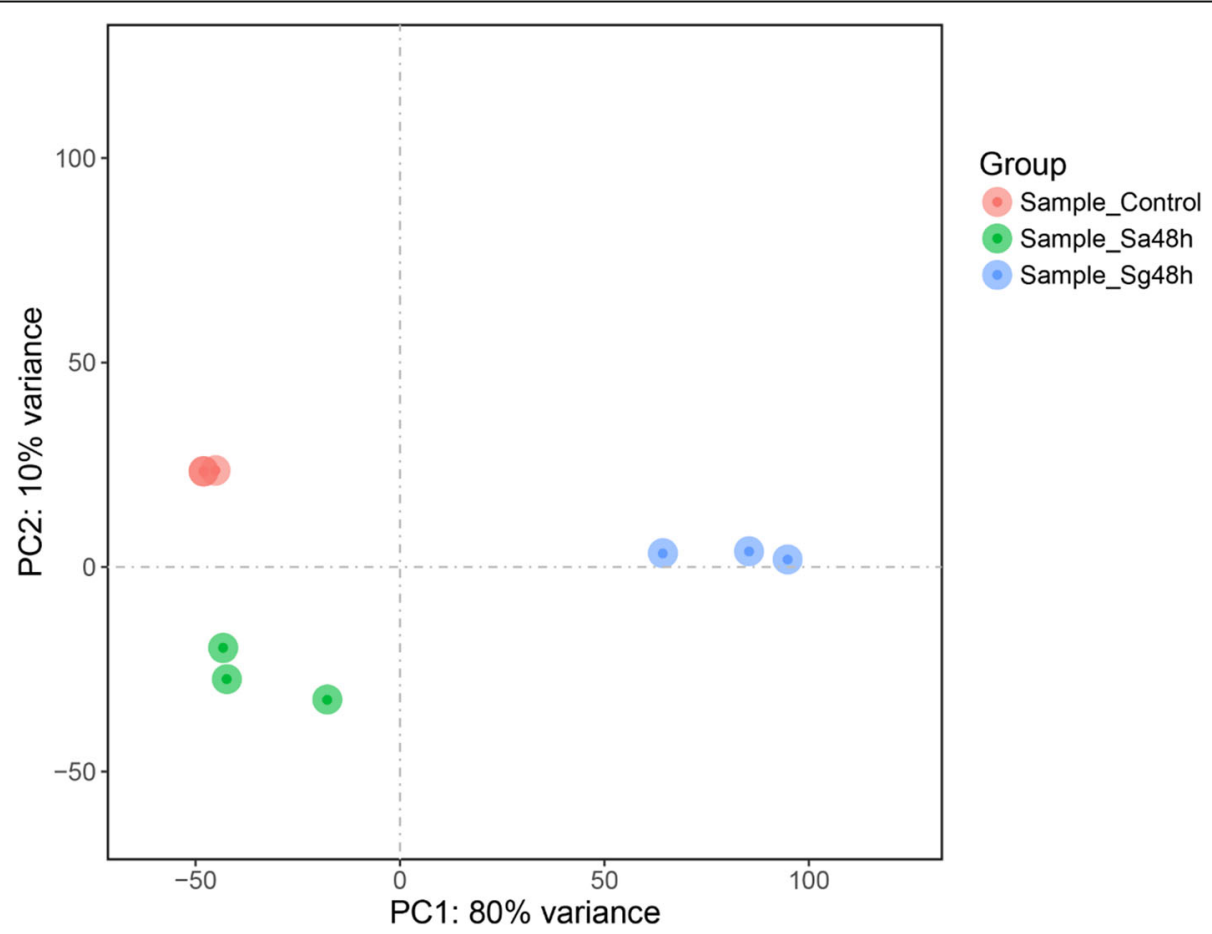

Fig. 3 Principal component analysis (PCA) plot on transcriptome data from control groups (red spots), wheat leaves infested with S. avenae for $48 \mathrm{~h}$ (Sa48h, green spots) and wheat leaves infested with S. graminum for $48 \mathrm{~h}$ (Sg48h, blue spots) 


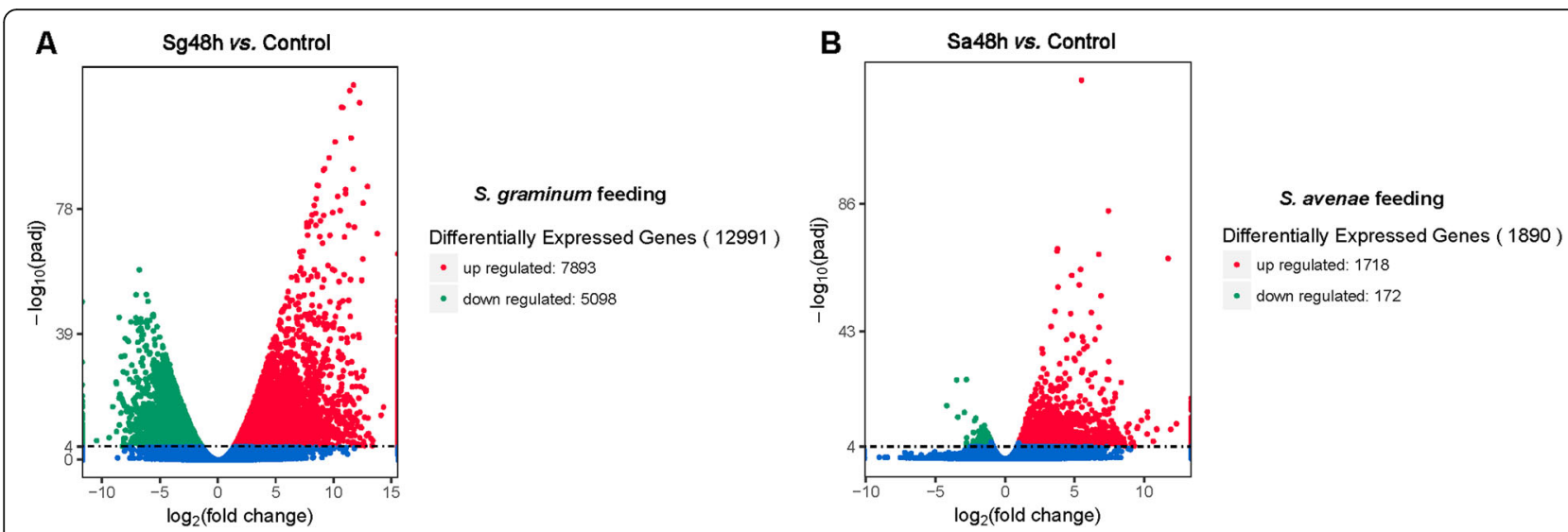

Fig. 4 Volcano plots of differentially expressed genes (DEGs) between the aphid-free wheat leaves and those infested with S. graminum (a) or S. avenae (b) for $48 \mathrm{~h}$. Each dot represents one gene with the $y$-axis showing $-\log _{10} q$ value and the $x$-axis showing $\log _{2}$ fold change, respectively. The red, green and blue dots represent the up-regulated DEGs, down-regulated DEGs (adjusted $p$ values $<0.00001,\left|\log _{2} F C\right| \geq 1$ ) and not significantly changed genes, respectively

All the DEGs were subjected to GO term and KEGG pathway analyses to identify the major DEG-associated metabolic pathways. The top 30 enriched GO terms and 20 most enriched KEGG pathways are shown in Fig. 6 and Figure S1 (Additional file 3). As shown in Fig. 6, within the biological process category, the DEGs induced by $S$. graminum were mainly enriched in the electron transport, small molecule metabolic process and carbohydrate metabolic process terms, and the DEGs induced by $S$. avenae were mainly enriched in protein phosphorylation, protein modification process and phosphorus metabolic process. Within the molecular function category, the largest proportion of DEGs induced by S. graminum was enriched in the catalytic activity and oxidoreductase activity terms, and the majority of the DEGs activated by $S$. avenae were enriched in the catalytic activity, protein kinase activity and phosphotransferase activity.
Transcripts related to photosynthesis, sucrose and starch metabolism and nitrogen metabolism

S. graminum feeding negatively affected the photosynthesis process of wheat, and many genes associated with light-harvesting and photosystem-associated genes, such as chlorophyll a-b binding proteins, ferrochelatase, and photosystem I and II proteins, were significantly downregulated (Table 2). The expression levels of ribulose bisphosphate carboxylase oxygenase (RuBisCO) and carbonic anhydrase genes with roles in the Calvin cycle were also significantly reduced after $S$. graminum feeding. However, few genes involved in photosynthesis were significantly regulated in the $S$. avenae-infested plants. The transcriptional profiles of some genes involved in sucrose and starch metabolism were also investigated. The sucrose synthase 3 gene, one trehalose-6-phosphate synthase gene and six beta-glucosidase genes were significantly downregulated in S. graminum- and S. avenae-

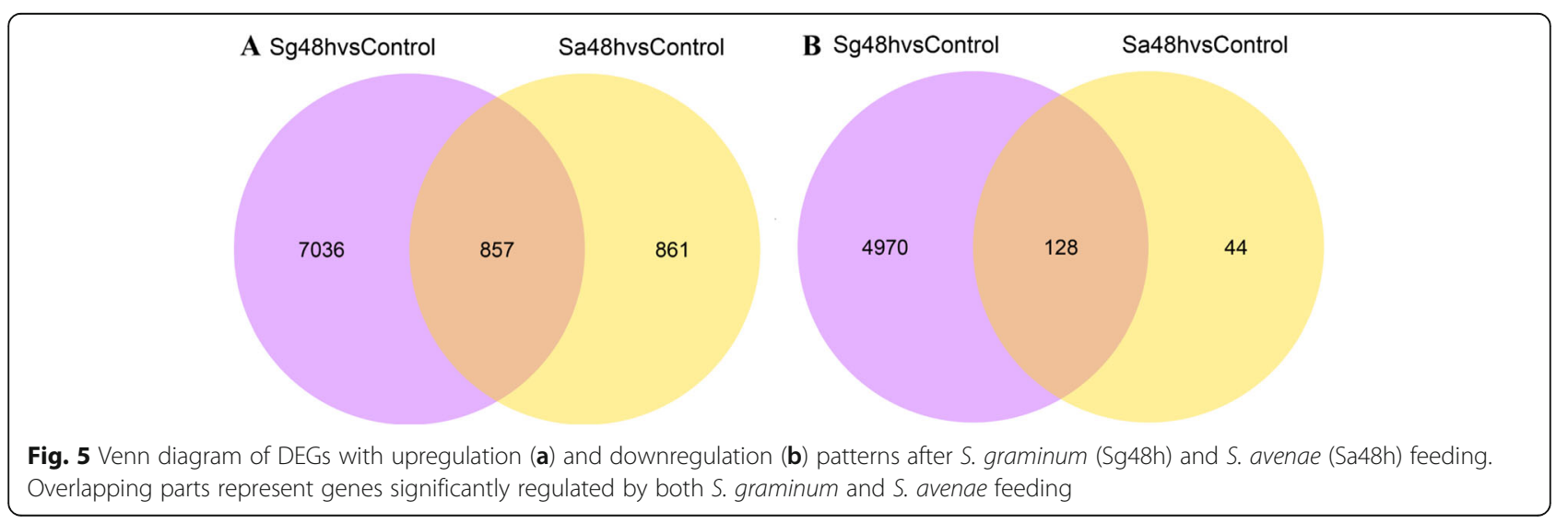




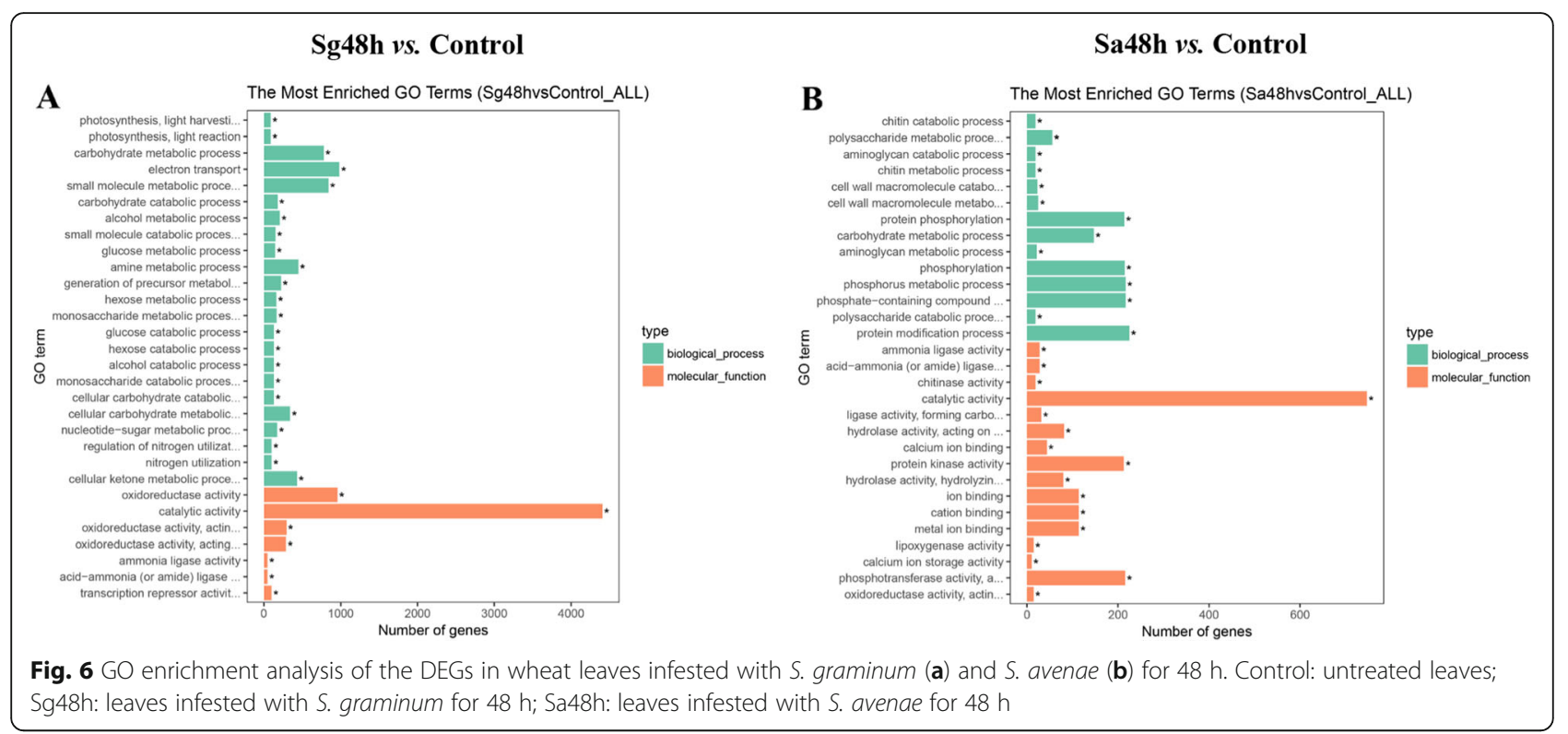

infested leaves, and the transcript levels of sucrose-phosphatase genes were significantly upregulated in wheat leaves infested with S. graminum and S. avenae. S. graminum feeding but not $S$. avenae feeding also significantly affected nitrogen metabolism. The transcript levels of nitrate reductase in the leaves were strongly downregulated by S. graminum, and glutamate dehydrogenase was significantly upregulated in wheat leaves infested with S. graminum. However, few genes involved in nitrogen metabolism were modulated by $S$. avenae feeding.

\section{Effects of S. graminum and S. avenae feeding on transcripts related to the SA, JA, and ET signalling pathways involved in plant defence}

To characterize how plant defence responses are modulated in response to $S$. graminum and $S$. avenae feeding, genes known to be involved in the SA, JA, and ET-defence pathways were examined [24]. The transcriptome data in Table 3 showed that 21 PAL genes involved in SA biosynthesis were significantly upregulated in response to $S$. graminum and $S$. avenae feeding. Furthermore, $13 P R$ genes responding to SA were significantly upregulated by S. graminum and S. avenae feeding. Additionally, under $S$. graminum and $S$. avenae feeding, one AOC (3.7-fold), three AOS (2.7- to 7.9-fold) and five LOX (2.8- to 8.8-fold) genes involved in JA biosynthesis were significantly upregulated, and the expression levels of three PI genes, which are JA-responsive defence genes, significantly increased. Two ACS (5.1 to 7.8-fold) and three ACO (4.0 to 5.0-fold) genes, which are involved in the ET signalling pathway, and 11 genes encoding ethylene-responsive transcription factors involved in ET biosynthesis were significantly upregulated in response to $S$. graminum feeding, but only one ACO (4.4-fold) gene and two genes encoding ethylene-responsive transcription factors (1.8-fold and induced) were upregulated after $S$. avenae feeding.

Although the transcript levels of some defence genes were significantly upregulated in response to both S. graminum and S. avenae feeding, a higher number of DEGs involved in SA-, JA-, and ET-mediated defence pathways were induced by $S$. graminum feeding than by $S$. avenae feeding (Additional file 4: Data S2). For example, $37 P R$ genes (downstream of SA) and 10 PI genes (downstream of JA) were significantly upregulated in response to $S$. graminum feeding, but only $17 P R$ genes and four $P I$ genes were significantly upregulated in response to $S$. avenae feeding. Additionally, S. graminum feeding induced greater fold changes in these two types of genes than $S$. avenae feeding, which indicated that the former triggered a stronger defence response than the latter.

\section{Effects of S. graminum feeding on transcripts associated with plant cell wall modification proteins (PCMDPs) in wheat leaves}

S. graminum feeding induced the expression of many genes encoding enzymatic or non-enzymatic proteins related to plant cell wall dynamics (Table 4). For example, the transcript levels of callose synthases were significantly upregulated after $S$. graminum and $S$. avenae feeding. The transcript levels of 19 PGs (1.92 to 6.45fold; induced) and four PEMs (3.37 to 3.97 -fold; induced) were significantly increased after $48 \mathrm{~h}$ of $S$. graminum feeding, but no PGs or PEMs were significantly induced in response to $S$. avenae feeding. Similarly, the transcript levels of six genes encoding beta-expansin, which is a non-enzymatic protein that plays important roles in cell wall loosening, were significantly upregulated (5.30 to 8.70-fold; induced) in wheat leaves infested 
Table 2 DEGs associated with primary plant metabolism in wheat leaves in response to S. graminum (Sg48h) and S. avenae (Sa48h) feeding

\begin{tabular}{|c|c|c|c|c|c|c|}
\hline \multirow[t]{2}{*}{ Pathways } & \multirow[t]{2}{*}{ Gene description } & \multirow[t]{2}{*}{ Gene ID } & \multicolumn{2}{|l|}{$\mathrm{Sg} 48 \mathrm{~h}$} & \multicolumn{2}{|l|}{ Sa $48 \mathrm{~h}$} \\
\hline & & & $\log _{2} F C$ & $q$-value & $\log _{2} F C$ & $q$-value \\
\hline \multirow[t]{35}{*}{ photosynthesis } & \multirow[t]{11}{*}{ Chlorophyll $a-b$ binding protein } & TraesCS5B02G462800 & -8.2468 & $4.71 \mathrm{E}-05$ & \multirow[t]{11}{*}{ / } & \\
\hline & & TraesCS5A02G454200 & -7.2799 & $4.32 \mathrm{E}-10$ & & \\
\hline & & TraesCS5B02G462900 & -7.4537 & $1.36 \mathrm{E}-05$ & & \\
\hline & & TraesCS1D02G411600 & -7.5869 & $1.42 \mathrm{E}-09$ & & \\
\hline & & TraesCS1A02G403800 & -7.1482 & $3.43 \mathrm{E}-28$ & & \\
\hline & & TraesCS6A02G094200 & -7.2817 & $3.20 \mathrm{E}-16$ & & \\
\hline & & TraesCS6A02G094500 & -7.038 & $3.66 \mathrm{E}-08$ & & \\
\hline & & TraesCS6D02G088800 & -6.9431 & 5.03E-06 & & \\
\hline & & TraesCS5D02G464700 & -6.8985 & $5.19 \mathrm{E}-11$ & & \\
\hline & & TraesCS5B02G463000 & -6.8386 & 3.60E-09 & & \\
\hline & & TraesCS6A02G094600 & -6.8352 & $1.98 \mathrm{E}-05$ & & \\
\hline & \multirow[t]{2}{*}{ Ferrochelatase } & TraesCS1A02G135700 & -1.6923 & 4.67E-06 & \multirow[t]{2}{*}{ / } & \\
\hline & & TraesCS1D02G131200 & -1.9256 & 1.97E-07 & & \\
\hline & \multirow[t]{6}{*}{ Photosystem I proteins } & TraesCS2D02G253200 & -4.3659 & $6.19 \mathrm{E}-31$ & \multirow[t]{6}{*}{ / } & \\
\hline & & TraesCS1B02G420100 & -3.9963 & 2.67E-25 & & \\
\hline & & TraesCS2A02G252600 & -3.9493 & $2.05 E-26$ & & \\
\hline & & TraesCS2B02G272300 & -3.8273 & $3.85 \mathrm{E}-25$ & & \\
\hline & & TraesCS1A02G392000 & -3.7654 & $3.25 \mathrm{E}-24$ & & \\
\hline & & TraesCS5A02G256900 & -3.7814 & $2.36 \mathrm{E}-24$ & & \\
\hline & \multirow[t]{6}{*}{ Photosystem II proteins } & TraesCS4A02G355600 & -3.9717 & 4.97E-07 & \multirow[t]{6}{*}{ / } & \\
\hline & & TraesCS5A02G386400 & -3.6099 & 6.97E-11 & & \\
\hline & & TraesCS7B02G215000 & -3.6384 & 2.27E-22 & & \\
\hline & & TraesCS7A02G314100 & -2.8644 & $3.79 \mathrm{E}-15$ & & \\
\hline & & TraesCS3B02G344200 & -2.9397 & $4.08 \mathrm{E}-16$ & & \\
\hline & & TraesCS7A02G314100 & -3.1573 & 5.97E-18 & & \\
\hline & \multirow[t]{3}{*}{ Photosynthetic NDH } & TraesCS6D02G287300 & -3.8223 & $1.11 \mathrm{E}-18$ & \multirow[t]{3}{*}{ / } & \\
\hline & & TraesCS6A02G308100 & -4.9624 & 1.33E-19 & & \\
\hline & & TraesCS4D02G273000 & -3.3857 & $1.73 \mathrm{E}-17$ & & \\
\hline & \multirow[t]{3}{*}{ Carbonic anhydrase } & TraesCS3A02G230000 & -6.5051 & $1.82 \mathrm{E}-15$ & \multirow[t]{3}{*}{ / } & \\
\hline & & TraesCS3B02G259300 & -6.7942 & 9.39E-14 & & \\
\hline & & TraesCS3D02G223300 & -6.4226 & 4.53E-12 & & \\
\hline & \multirow[t]{4}{*}{ RuBisCO } & TraesCS2D02G065400 & -7.0835 & $1.64 \mathrm{E}-06$ & \multirow[t]{4}{*}{ / } & \\
\hline & & TraesCS2D02G065300 & -6.3924 & $6.01 \mathrm{E}-08$ & & \\
\hline & & TraesCS2D02G065100 & -6.1885 & $6.39 \mathrm{E}-06$ & & \\
\hline & & TraesCS2D02G065200 & -5.3796 & $6.33 \mathrm{E}-06$ & & \\
\hline \multirow[t]{6}{*}{ sucrose and starch metabolisms } & Trehalose-6-phosphate synthase & TraesCS1A02G339300 & -2.2863 & $1.92 \mathrm{E}-09$ & -1.1024 & $6.64 \mathrm{E}-05$ \\
\hline & sucrose synthase 3 & TraesCS2A02G168200 & -3.4137 & 1.60E-09 & -2.3629 & 7.91E-05 \\
\hline & Sucrose-phosphatase & TraesCS1B02G107600 & 4.0937 & 4.86E-10 & 3.0633 & 8.93E-09 \\
\hline & \multirow[t]{3}{*}{ Beta-glucosidase } & TraesCS2B02G401500 & -2.1186 & $1.62 \mathrm{E}-09$ & -1.602 & 3.62E-07 \\
\hline & & TraesCS2D02G381000 & -2.1015 & 1.30E-09 & -1.4152 & 3.85E-10 \\
\hline & & TraesCS3D02G440200 & -6.7792 & $7.04 \mathrm{E}-60$ & -1.0065 & 2.14E-06 \\
\hline nitrogen metabolisms & Nitrate reductase & TraesCS6B02G356800 & Induced & $6.33 \mathrm{E}-16$ & / & \\
\hline
\end{tabular}


Table 2 DEGs associated with primary plant metabolism in wheat leaves in response to S. graminum (Sg48h) and S. avenae (Sa48h) feeding (Continued)

\begin{tabular}{|c|c|c|c|c|c|c|}
\hline \multirow[t]{2}{*}{ Pathways } & \multirow[t]{2}{*}{ Gene description } & \multirow[t]{2}{*}{ Gene ID } & \multicolumn{2}{|l|}{$\mathrm{Sg} 48 \mathrm{~h}$} & \multicolumn{2}{|l|}{ Sa $48 \mathrm{~h}$} \\
\hline & & & $\log _{2} F C$ & q-value & $\log _{2} F C$ & $q$-value \\
\hline & & TraesCS6D02G306000 & Induced & $9.79 \mathrm{E}-17$ & & \\
\hline & & TraesCS6A02G326200 & Induced & $3.11 \mathrm{E}-10$ & & \\
\hline & Glutamine synthetase & TraesCS2A02G500400 & -3.0056 & $8.28 \mathrm{E}-07$ & / & \\
\hline & & TraesCS2B02G528300 & -3.0711 & $6.95 \mathrm{E}-09$ & & \\
\hline & & TraesCS2D02G500600 & -3.7958 & $5.12 \mathrm{E}-24$ & & \\
\hline & & TraesCS6B02G327500 & 1.9819 & $1.68 \mathrm{E}-08$ & & \\
\hline & Glutamate dehydrogenase & TraesCS2B02G409300 & 6.9374 & 3.09E-08 & / & \\
\hline & & TraesCS2A02G389900 & 6.5323 & $1.14 \mathrm{E}-06$ & & \\
\hline & Cysteine synthase & TraesCS3A02G338600 & -2.8015 & $5.21 \mathrm{E}-06$ & / & \\
\hline & & TraesCS3B02G370200 & -2.9708 & 1.56E-05 & & \\
\hline & & TraesCS4A02G401600 & -2.4908 & 3.85E-09 & & \\
\hline & & TraesCS6B02G217200 & 3.9529 & 2.04E-07 & & \\
\hline
\end{tabular}

"I" indicates no significant differences between aphid infested and control groups

with S. graminum, but no expansin genes were significantly regulated after $48 \mathrm{~h}$ of $S$. avenae feeding.

\section{Effects of S. graminum and S. avenae feeding on the transcript levels and activities of antioxidant enzymes involved in ROS scavenging in wheat leaves}

In plants, herbivore attacks usually trigger oxidative responses [25]. Plants possess a battery of ROS scavengers, such as POD, SOD, and CAT enzymes, and these enzymes can protect cells from oxidative damage [26]. As shown in Fig. 7, 74 PODs were significantly up- or down- regulated in response to $S$. graminum feeding, and 66 of these PODs were significantly upregulated. However, only 15 PODs were significantly upregulated by $S$. avenae feeding. Similarly, the expression levels of $12 A P x$ genes were significantly modulated by S. graminum feeding, but the expression levels of only two $A P x$ genes were significantly affected by $S$. avenae feeding. Additionally, five $C A T$, eight $S O D$ and seven glutathione peroxidase (GPX) genes were significantly regulated by $S$. graminum feeding, but not by $S$. avenae feeding (Additional file 5: Data S3). The increased number of ROS scavengers induced by $S$. graminum feeding suggested that $S$. graminum feeding induces stronger oxidative stress in wheat leaves than $S$. avenae.

Compared with the control, the activity of POD was significantly increased after $24 \mathrm{~h}\left(\mathrm{t}_{4}=-4.387, P=0.012\right)$ of $S$. graminum feeding and reached a peak at $48 \mathrm{~h}\left(\mathrm{t}_{4}=-\right.$ 9.981, $P=0.001$ ) (Fig. 8). The activity of POD in leaves was also significantly increased $72 \mathrm{~h}$ after $S$. avenae feeding $\left(t_{4}=-3.353, P=0.028\right)$. Furthermore, the activities of SOD, CAT and APx were significantly increased after $24 \mathrm{~h}$ of $S$. graminum feeding $\left(\mathrm{t}_{4}=-12.295, P<0.001 ; \mathrm{t}_{4}=-\right.$ 2.789, $\left.P=0.049 ; \mathrm{t}_{4}=-7.761, P=0.001\right)$, whereas $S$. avenae feeding had no significant effects on the activities of SOD, CAT and APx $\left(\mathrm{t}_{4}=-1.560, P=0.194 ; \mathrm{t}_{4}=-0.600, P=\right.$ $\left.0.581 ; \mathrm{t}_{4}=0.048, P=0.964\right)$.

\section{Cytological examination of callose deposition and ROS accumulation in aphid-infested leaves}

To detect whether callose was deposited at the feeding sites, aphid-infested leaves were stained with aniline blue. As shown in Fig. 9, no callose deposits were observed in vascular tissues without aphid infestation (Fig. 9a). However, in S. avenae- and S. graminuminfested tissues, callose deposits were clearly detected as bright blue fluorescence directly at the feeding sites (Fig. 9b and c).

$\mathrm{H}_{2} \mathrm{O}_{2}$ accumulation has been shown to be induced by wounding and by pathogen and herbivore attacks in plants and is involved in plant defence responses as a signal molecule [27]. To record the accumulation of $\mathrm{H}_{2} \mathrm{O}_{2}$ after aphid infestation, $S$. avenae- and S. graminum-infested leaves were examined after cytological staining with $\mathrm{DAB}$, which was used to detect the production of $\mathrm{H}_{2} \mathrm{O}_{2}$. As shown in Fig. 9d-e, no obvious $\mathrm{DAB}$ staining was observed in the non-infested leaves, and a small brown-stained area was detected in the wheat leaves after $S$. avenae feeing. However, $\mathrm{H}_{2} \mathrm{O}_{2}$ was clearly detected in the areas of $S$. graminum feeding, which indicated that $S$. graminum feeding induced a massive accumulation of $\mathrm{H}_{2} \mathrm{O}_{2}$ (Fig. 9f). The $\mathrm{H}_{2} \mathrm{O}_{2}$ contents in the feeding sites of wheat leaves after $S$. avenae and S. graminum infestations were also examined. As indicated in Fig. 10, the concentration of $\mathrm{H}_{2} \mathrm{O}_{2}$ in wheat leaves infested with $S$. graminum $(143.19 \pm 31.15 \mu \mathrm{mol}$ $\left.\mathrm{g}^{-1} \mathrm{FW} ; \mathrm{F}_{2,6}=7.345 ; P=0.024\right)$ was significantly higher than that in $S$. avenae-infested and control leaves. In 
Table 3 DEGs involved in SA, JA and ET- dependent defense pathways in response to S. graminum (Sg48h) and S. avenae (Sa48h) feeding

\begin{tabular}{|c|c|c|c|c|c|c|}
\hline \multirow[t]{2}{*}{ Pathways } & \multirow[t]{2}{*}{ Gene description } & \multirow[t]{2}{*}{ Gene ID } & \multicolumn{2}{|l|}{$\mathrm{Sg} 48 \mathrm{~h}$} & \multicolumn{2}{|l|}{ Sa $48 \mathrm{~h}$} \\
\hline & & & $\log _{2} F C$ & $q$-value & $\log _{2} F C$ & $q$-value \\
\hline \multirow[t]{34}{*}{ SA-defense pathway } & \multirow[t]{21}{*}{ Phenylalanine ammonia-lyase (PAL) } & TraesCS1A02G037700 & Induced & 4.21E-06 & Induced & $1.22 \mathrm{E}-06$ \\
\hline & & TraesCS1B02G122800 & 6.71 & $7.58 \mathrm{E}-09$ & 6.98 & $1.38 \mathrm{E}-06$ \\
\hline & & TraesCS1D02G039300 & 6.76 & $2.51 \mathrm{E}-23$ & 7.13 & $4.31 \mathrm{E}-06$ \\
\hline & & TraesCS1D02G039400 & 6.45 & $3.28 \mathrm{E}-07$ & 7.09 & $7.79 \mathrm{E}-07$ \\
\hline & & TraesCS1D02G103500 & 5.75 & $1.54 \mathrm{E}-05$ & 5.88 & $6.89 \mathrm{E}-07$ \\
\hline & & TraesCS2A02G196400 & 5.33 & 1.18E-05 & 5.17 & $1.62 \mathrm{E}-06$ \\
\hline & & TraesCS2A02G196700 & Induced & $3.75 \mathrm{E}-20$ & Induced & 2.47E-07 \\
\hline & & TraesCS2A02G381000 & 6.24 & $2.33 \mathrm{E}-11$ & 5.90 & $2.08 \mathrm{E}-06$ \\
\hline & & TraesCS2A02G381100 & 5.14 & $2.20 \mathrm{E}-16$ & 4.33 & $3.11 \mathrm{E}-05$ \\
\hline & & TraesCS2B02G224300 & 7.90 & 9.99E-09 & 8.22 & 2.83E-07 \\
\hline & & TraesCS2B02G398100 & 3.39 & 2.81E-05 & 4.57 & $7.02 \mathrm{E}-12$ \\
\hline & & TraesCS2B02G398200 & 5.80 & $2.59 \mathrm{E}-17$ & 5.04 & $6.19 \mathrm{E}-06$ \\
\hline & & TraesCS2B02G398400 & 4.79 & $6.14 \mathrm{E}-10$ & 4.80 & $1.40 \mathrm{E}-05$ \\
\hline & & TraesCS2D02G204400 & 9.53 & 4.40E-07 & 8.44 & $3.35 \mathrm{E}-07$ \\
\hline & & TraesCS2D02G377200 & 4.54 & 7.91E-23 & 4.32 & 4.15E-07 \\
\hline & & TraesCS2D02G377500 & 5.51 & 4.27E-26 & 4.43 & $5.23 \mathrm{E}-05$ \\
\hline & & TraesCS4A02G401300 & 9.34 & $2.75 \mathrm{E}-13$ & 9.48 & $9.50 \mathrm{E}-11$ \\
\hline & & TraesCS5B02G468300 & 5.52 & 2.74E-09 & 7.64 & $2.18 \mathrm{E}-11$ \\
\hline & & TraesCS5B02G468400 & 5.24 & $5.32 \mathrm{E}-06$ & 6.35 & $1.20 \mathrm{E}-07$ \\
\hline & & TraesCS6A02G222700 & 4.72 & 8.97E-06 & 6.48 & $1.28 \mathrm{E}-14$ \\
\hline & & TraesCS6B02G258600 & 6.08 & $8.58 \mathrm{E}-11$ & 5.77 & $5.11 \mathrm{E}-07$ \\
\hline & \multirow[t]{13}{*}{ Pathogenesis-related protein (PR protein) } & TraesCS1A02G355300 & 7.37 & $1.52 \mathrm{E}-65$ & 5.43 & $1.90 \mathrm{E}-64$ \\
\hline & & TraesCS1B02G366300 & Induced & $1.05 \mathrm{E}-15$ & Induced & $8.39 \mathrm{E}-05$ \\
\hline & & TraesCS2D02G317800 & 7.69 & 1.16E-15 & 8.12 & 4.17E-09 \\
\hline & & TraesCS3A02G480400 & 10.19 & 5.99E-14 & 5.34 & $1.02 \mathrm{E}-12$ \\
\hline & & TraesCS3B02G525000 & 10.30 & $1.40 \mathrm{E}-12$ & 6.20 & $5.96 \mathrm{E}-29$ \\
\hline & & TraesCS3D02G475200 & 8.93 & 4.04E-15 & 5.55 & $3.58 \mathrm{E}-06$ \\
\hline & & TraesCS4D02G015800 & 7.02 & $6.75 \mathrm{E}-26$ & 3.27 & 3.39E-05 \\
\hline & & TraesCS5A02G018200 & 5.72 & $7.89 \mathrm{E}-17$ & 4.26 & 4.95E-26 \\
\hline & & TraesCS5A02G183300 & 9.92 & 7.93E-60 & 5.56 & $1.27 \mathrm{E}-30$ \\
\hline & & TraesCS5A02G439800 & 12.67 & 7.78E-14 & 7.21 & $1.44-09$ \\
\hline & & TraesCS5B02G181500 & 9.93 & $1.35 \mathrm{E}-77$ & 5.67 & $1.11 \mathrm{E}-37$ \\
\hline & & TraesCS5B02G442700 & 13.32 & 4.11E-05 & 7.26 & 3.37E-06 \\
\hline & & TraesCS7D02G161200 & 10.06 & $2.20 \mathrm{E}-20$ & 6.19 & $5.78 \mathrm{E}-50$ \\
\hline \multirow[t]{8}{*}{ JA-defense pathway } & \multirow[t]{3}{*}{ Allene oxide synthase (AOS) } & TraesCS4A02G061900 & 7.09 & $3.88 \mathrm{E}-13$ & 6.96 & $5.85 \mathrm{E}-08$ \\
\hline & & TraesCS4B02G237600 & 5.54 & 2.23E-06 & 6.23 & $6.93 \mathrm{E}-17$ \\
\hline & & TraesCS4D02G238800 & 2.77 & 1.19E-12 & 4.42 & 2.27E-08 \\
\hline & Allene oxide cyclase (AOC) & TraesCS6D02G314300 & 3.70 & $5.68 \mathrm{E}-05$ & 2.06 & $2.39 \mathrm{E}-05$ \\
\hline & \multirow[t]{3}{*}{ Lipoxygenase (LOX) } & TraesCS4B02G037700 & 2.84 & $8.85 \mathrm{E}-15$ & 3.44 & $2.11 \mathrm{E}-09$ \\
\hline & & TraesCS4B02G037800 & 3.75 & $2.55 \mathrm{E}-22$ & 2.79 & 2.17E-06 \\
\hline & & TraesCS4B02G037900 & 4.70 & $8.54 \mathrm{E}-36$ & 4.47 & 8.67E-08 \\
\hline & Proteinase inhibitors (PIs) & TraesCS3A02G046100 & 8.32 & $1.53 \mathrm{E}-29$ & 4.71 & 7.90E-09 \\
\hline
\end{tabular}


Table 3 DEGs involved in SA, JA and ET- dependent defense pathways in response to S. graminum (Sg48h) and S. avenae (Sa48h) feeding (Continued)

\begin{tabular}{|c|c|c|c|c|c|c|}
\hline \multirow[t]{2}{*}{ Pathways } & \multirow[t]{2}{*}{ Gene description } & \multirow[t]{2}{*}{ Gene ID } & \multicolumn{2}{|l|}{$\mathrm{Sg} 48 \mathrm{~h}$} & \multicolumn{2}{|l|}{ Sa $48 \mathrm{~h}$} \\
\hline & & & $\log _{2} F C$ & $q$-value & $\log _{2} F C$ & $q$-value \\
\hline & & TraesCS3B02G038700 & 8.81 & 2.09E-21 & 5.85 & $1.51 \mathrm{E}-15$ \\
\hline & & TraesCS3D02G034200 & Induced & $2.08 \mathrm{E}-10$ & Induced & $3.50 \mathrm{E}-09$ \\
\hline \multirow[t]{15}{*}{ ET-signaling pathway } & 1-aminocyclopropane-1-carboxylate synthase (ACS) & TraesCS2B02G414800 & 7.84 & $5.12 \mathrm{E}-08$ & / & / \\
\hline & & TraesCS2D02G394200 & 5.10 & 3.76-E05 & & \\
\hline & 1-aminocyclopropane-1-carboxylate oxidase 1 (ACO) & TraesCS6B02G355900 & 4.43 & 4.11-E09 & / & / \\
\hline & & TraesCS6A02G325700 & 4.03 & 6.84E-07 & & \\
\hline & & TraesCS6B02G356200 & 5.04 & $2.31 \mathrm{E}-11$ & & \\
\hline & & TraesCS4A02G109200 & / & / & 4.39 & 8.67E-05 \\
\hline & ET-responsive transcription factors & TraesCS6B02G281000 & Induced & $1.18 \mathrm{E}-08$ & Induced & 4.12E-05 \\
\hline & & TraesCS4A02G326400 & Induced & $6.85 E-26$ & / & / \\
\hline & & TraesCS4D02G313400 & Induced & 2.10E-05 & & \\
\hline & & TraesCS1B02G282300 & Induced & 7.57E-05 & & \\
\hline & & TraesCS2D02G414300 & Induced & $1.72 \mathrm{E}-06$ & & \\
\hline & ET-insensitive protein & TraesCS5A02G547500 & -2.1748 & 1.73E-08 & -1.2853 & 3.07E-07 \\
\hline & & TraesCS4A02G275600 & -3.3408 & 1.20E-09 & / & / \\
\hline & & TraesCS4D02G036000 & -3.5712 & $4.72 \mathrm{E}-16$ & & \\
\hline & & TraesCS7B02G145400 & -2.3144 & $1.46 \mathrm{E}-07$ & & \\
\hline
\end{tabular}

"I" indicates no significant differences between aphid-infested and control groups

contrast, $S$. avenae feeding had no significant effects on the $\mathrm{H}_{2} \mathrm{O}_{2}$ content compared with the control (Fig. 10). The changes in the $\mathrm{H}_{2} \mathrm{O}_{2}$ content in response to aphid feeding were consistent with the $\mathrm{DAB}$ staining results.

\section{Scavenging of $\mathrm{H}_{2} \mathrm{O}_{2}$ using DMTU reduces S. graminum feeding-induced damage on wheat leaves}

To further investigate the role of $\mathrm{H}_{2} \mathrm{O}_{2}$ accumulation on the damage induced by $S$. graminum feeding, wheat seedlings infested with aphids were treated with $5 \mathrm{mM}$ DMTU (an $\mathrm{H}_{2} \mathrm{O}_{2}$ scavenger). The DAB staining results shown in Fig. 11 demonstrated that DMTU treatment inhibited the $S$. graminum feeding-induced production of $\mathrm{H}_{2} \mathrm{O}_{2}$ in wheat leaves and the symptoms of damage in wheat leaves caused by $S$. graminum feeding. The delayed fluorescence and chlorophyll content were also assessed, and the results showed that the DMTU-treated infested leaves showed decreased chlorophyll degradation and that the chlorophyll content in the DMTUtreated leaves was significantly higher than that in the non-DMTU-treated leaves infested with $S$. graminum $\left(\mathrm{F}_{2,6}=13.93, P=0.0056\right)$.

\section{Discussion}

A previous study showed that S. graminum feeding led to obvious feeding damage and the loss of chlorophyll in aphid-susceptible winter wheat accession Beijing 837 [18]. Similarly, serious chlorosis symptoms were observed on another winter wheat accession, Zhongmai 175 , after 48 h of S. graminum feeding in this study, and this effect was also accompanied by a significant reduction in the total chlorophyll content of the wheat leaves, further demonstrating the phytotoxic effects of S. graminum on susceptible wheat plants. To further compare the similarities and differences between the responses to $S$. graminum and $S$. avenae feeding at the molecular level, a comparative transcriptome analysis of wheat leaves after aphid feeding was performed. We found that more than 20,000 genes were significantly regulated in wheat infested with S. graminum, but only 1700 genes were significantly modulated after $48 \mathrm{~h}$ of $S$. avenae feeding, which indicated that the physiological changes induced by $S$. graminum are notably different from those induced by $S$. avenae and that various metabolic pathways are involved in the development of damage caused by $S$. graminum feeding. Moreover, many genes involved in plant photosynthesis were strongly downregulated after S. graminum feeding, and this finding provides molecular evidence showing that chlorosis is induced by $S$. graminum.

\section{S. graminum feeding induces stronger plant defence responses than $S$. avenae}

Piercing-sucking hemipteran insects, such as aphids and whiteflies, mainly induce SA-mediated defence signal pathways [28, 29]. However, some studies have also 
Table 4 Expression levels of plant cell wall-modifying proteins in response to S. graminum (Sg48h) and S. avenae (Sa48h) feeding in wheat leaves

\begin{tabular}{|c|c|c|c|c|c|}
\hline \multirow[t]{2}{*}{ Gene description } & \multirow[t]{2}{*}{ Gene ID } & \multicolumn{2}{|l|}{ Sg48h } & \multicolumn{2}{|l|}{ Sa48h } \\
\hline & & $\log _{2} F C$ & $q$ value & $\log _{2} F C$ & $q$ value \\
\hline \multirow[t]{2}{*}{ Callose synthases } & TraesCS2D02G600000 & 2.5134 & $3.11 \mathrm{E}-05$ & 3.1012 & $1.02 \mathrm{E}-05$ \\
\hline & TraesCSU02G021500 & 3.1218 & 4.49E-05 & 4.849 & $1.25 \mathrm{E}-12$ \\
\hline \multirow[t]{4}{*}{ Pectin acetylesterase (PAE) } & TraesCS2A02G377100 & 7.2447 & $6.75 \mathrm{E}-10$ & / & / \\
\hline & TraesCS5A02G486000 & -1.8876 & $3.22 \mathrm{E}-06$ & / & / \\
\hline & TraesCS2B02G178100 & / & / & 2.6875 & 2.00E-10 \\
\hline & TraesCS2D02G158400 & / & / & 2.5945 & 2.76E-08 \\
\hline \multirow[t]{19}{*}{ Polygalacturonase (PG) } & TraesCS1A02G311100 & 3.10 & $1.01 \mathrm{E}-14$ & / & \\
\hline & TraesCS1A02G364800 & 6.45 & 2.20E-05 & & \\
\hline & TraesCS1D02G311000 & 2.20 & 2.94E-07 & & \\
\hline & TraesCS2D02G421700 & 5.18 & 5.23E-05 & & \\
\hline & TraesCS3B02G258600 & 3.67 & $4.23 \mathrm{E}-05$ & & \\
\hline & TraesCS3D02G223900 & 4.70 & $2.26 \mathrm{E}-09$ & & \\
\hline & TraesCS4B02G086500 & 3.81 & $8.56 \mathrm{E}-21$ & & \\
\hline & TraesCS5A02G212900 & 2.68 & $1.42 \mathrm{E}-08$ & & \\
\hline & TraesCS5A02G459000 & Induced & $9.07 \mathrm{E}-06$ & & \\
\hline & TraesCS5A02G513600 & 3.75 & $3.75 \mathrm{E}-15$ & & \\
\hline & TraesCS5B02G210900 & 2.03 & $6.33 \mathrm{E}-06$ & & \\
\hline & TraesCS5B02G468700 & Induced & $1.08 \mathrm{E}-09$ & & \\
\hline & TraesCS5D02G102900 & 3.08 & 4.19E-11 & & \\
\hline & TraesCS5D02G219200 & 2.02 & $1.29 \mathrm{E}-06$ & & \\
\hline & TraesCS5D02G470100 & Induced & $1.03 \mathrm{E}-07$ & & \\
\hline & TraesCS6A02G084500 & Induced & $2.92 \mathrm{E}-06$ & & \\
\hline & TraesCS6B02G118000 & Induced & $2.39 \mathrm{E}-05$ & & \\
\hline & TraesCS7D02G326800 & 6.27 & 5.33E-09 & & \\
\hline & TraesCS1B02G322500 & 1.92 & $8.26 \mathrm{E}-05$ & & \\
\hline \multirow[t]{4}{*}{ Pectinesterase (PEM) } & Novel04561 & 3.37 & 4.08E-09 & / & \\
\hline & TraesCS1A02G263900 & Induced & $3.93 \mathrm{E}-38$ & & \\
\hline & TraesCS1D02G264000 & Induced & $5.08 \mathrm{E}-07$ & & \\
\hline & TraesCS2A02G173200 & 3.97 & $1.76 \mathrm{E}-09$ & & \\
\hline \multirow[t]{7}{*}{ Beta-expansin (EXP) } & TraesCS1A02G212300 & 7.17 & $2.96 \mathrm{E}-10$ & / & \\
\hline & TraesCS1B02G225700 & -4.59 & 4.09E-09 & & \\
\hline & TraesCS1B02G225900 & 5.30 & $6.23 \mathrm{E}-06$ & & \\
\hline & TraesCS1D02G215300 & 6.65 & $5.54 \mathrm{E}-13$ & & \\
\hline & TraesCS3A02G480000 & 8.70 & 2.20E-07 & & \\
\hline & TraesCS3B02G524500 & 8.61 & $1.25 \mathrm{E}-06$ & & \\
\hline & TraesCS5A02G073100 & Induced & 5.03E-08 & & \\
\hline
\end{tabular}

"I" indicates no significant differences between aphid-infested and control groups

demonstrated that genes involved in both the JA and SA defence response pathways, such as LOX, PIs, PAL, and $P R 1$, are significantly upregulated in response to aphid feeding $[20,30,31]$. Similarly, we found that both $S$. avenae and $S$. graminum feeding significantly increased the expression levels of genes related to the SA, JA and ET signalling pathways. Plant defence responses activated by aphids are closely associated with the plant species, aphid density and infestation time [32, 33]. In the future, we will further identify the wheat defence responses induced by $S$. graminum and S. avenae feeding under various aphid densities and feeding periods. Although both 


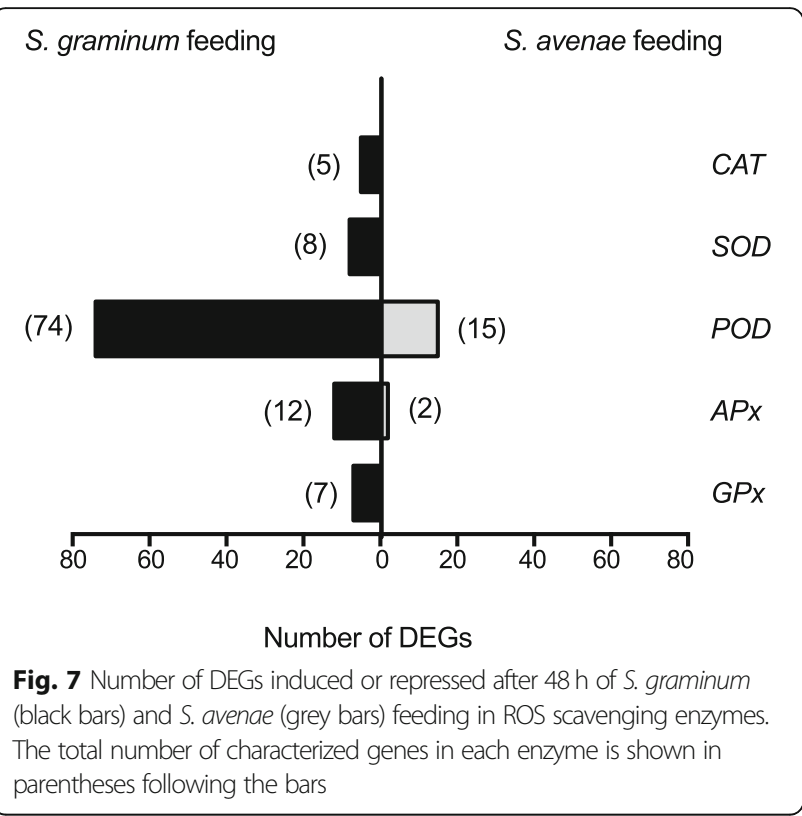

of these cereal aphids induced both SA-, JA- and ETdependent defence pathways, S. graminum feeding induced the expression of more genes involved in plant defence pathways in this study. For example, 30 PAL genes were upregulated in response to $S$. graminum feeding, but only four $P A L$ genes were upregulated in response to $S$. avenae feeding. The transcript levels of five ET-responsive genes were upregulated in response to S. graminum, but only one was modulated in response to $S$. avenae feeding. Zhang et al. demonstrated that the fold changes in the expression levels of $P R$ genes and the SA contents in wheat leaves were significantly greater after $S$. graminum feeding than after S. avenae feeding [22]. Argandoña et al. also suggested that $S$. graminum induced more ethylene production than the non-phytotoxic aphid Rhopalosiphum padi [34]. The stronger defence responses activated by $S$. graminum feeding than by $S$. avenae feeding might be responsible for the induction of chlorosis in wheat.

\section{Genes encoding plant cell-modifying proteins are significantly upregulated in response to $S$. graminum feeding}

Multiple modifications can be triggered in cell walls in response to microbial and insect attack [35]. Callose deposition in crop plants is observed in response to

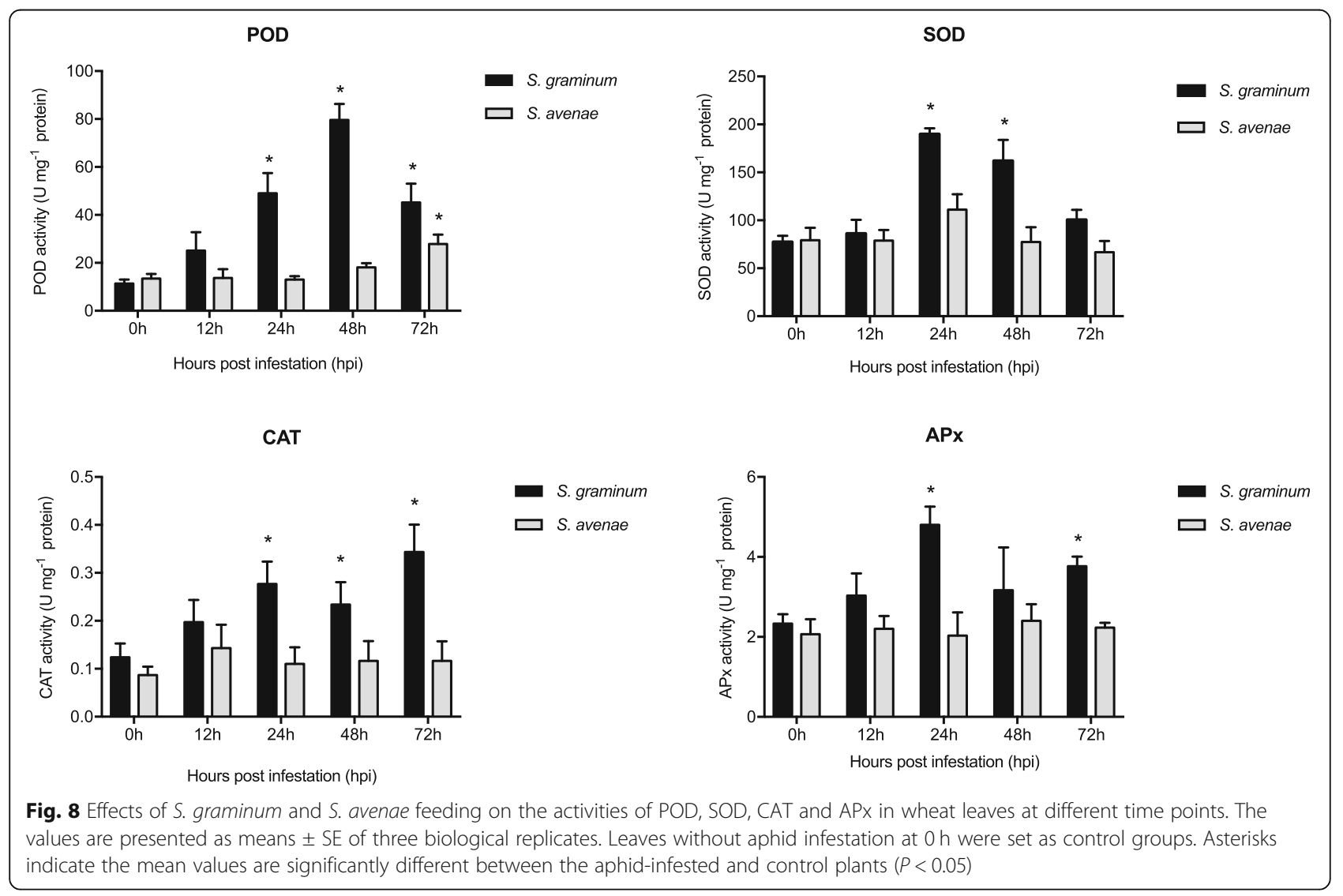



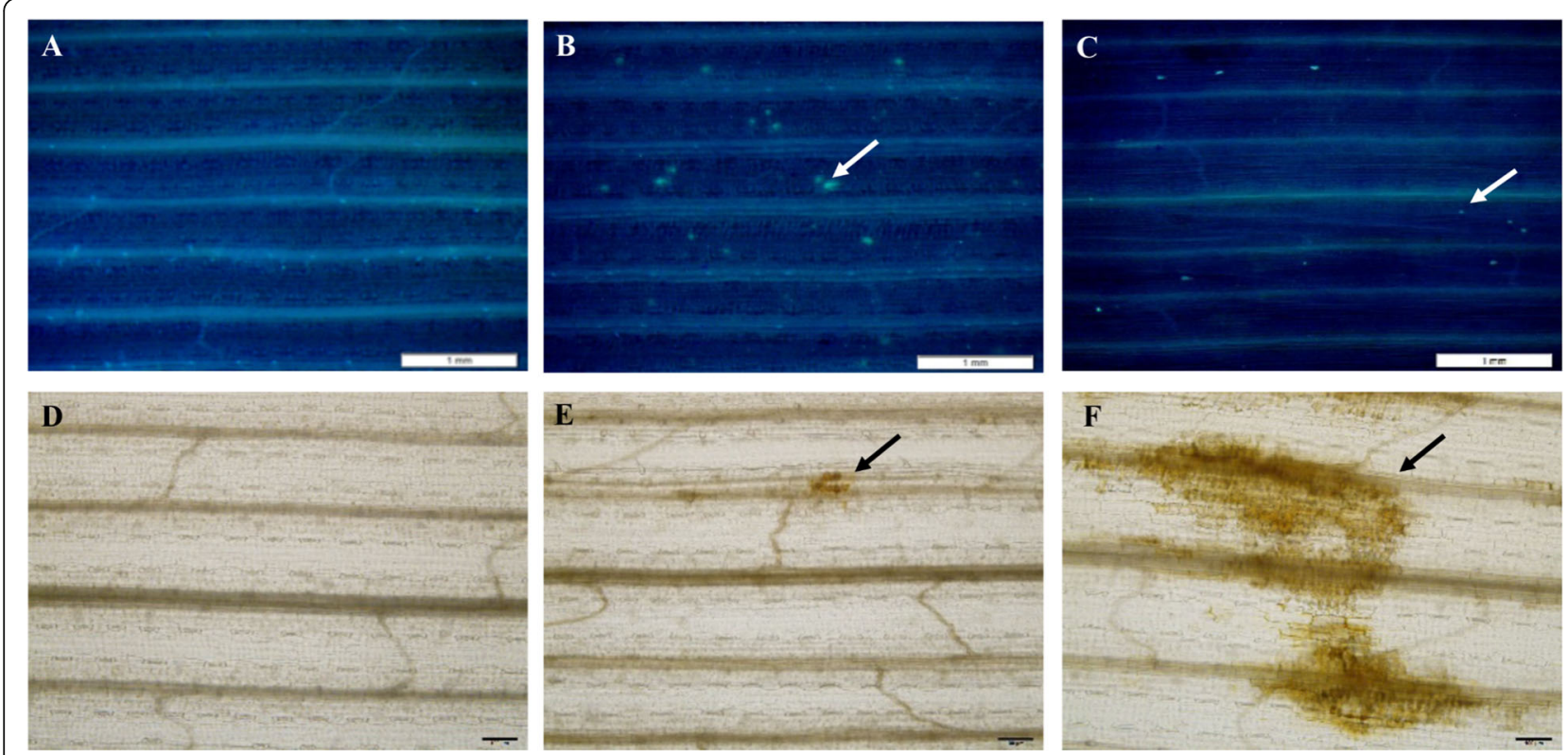

Fig. 9 Cytological examination of aphid-infested wheat leaves for $\mathrm{H}_{2} \mathrm{O}_{2}$ accumulation and callose deposition. Experiments were performed with three biological replicates with similar results, and representative results from one replicate are shown. a-c: aniline blue staining for callose deposition (scale bar $=1 \mathrm{~mm}$ ). d-f: DAB staining to detect $\mathrm{H}_{2} \mathrm{O}_{2}$ (scale bar $\left.=100 \mu \mathrm{m}\right)$. Untreated control leaves (a and $\left.\mathbf{d}\right)$; S. avenae-infested leaves (b and e); . graminum-infested leaves (c and $\mathbf{f}$ )

biotrophic fungal infection at papillae sites and in sieve elements in response to aphids [36, 37]. It has been proposed that callose deposition impedes fungal attacks at the sites of attempted penetration in epidermal cells and thereby supports pathogen resistance [38]. The transcript levels of callose synthases were significantly upregulated in response to $S$. graminum and $S$. avenae feeding. Obvious callose accumulation was also observed in wheat leaves after S. avenae and S. graminum feeding. However, the role of callose in aphid-plant interactions remains unknown. It has been hypothesized that callose

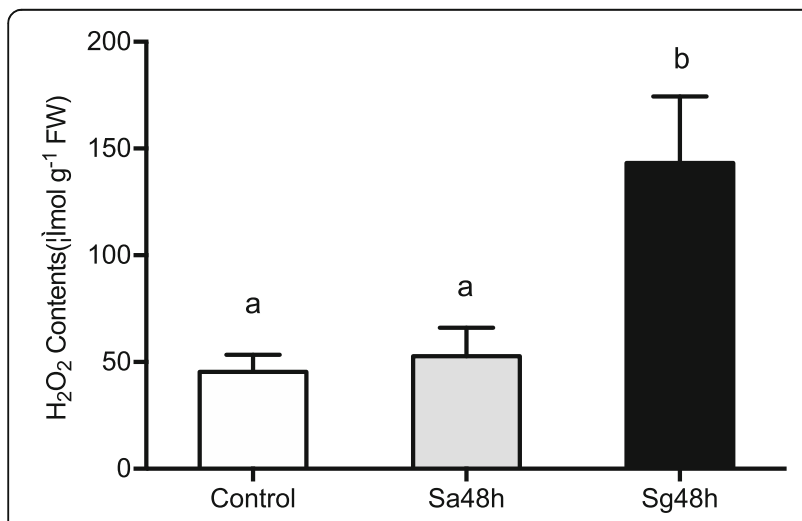

Fig. 10 Content of $\mathrm{H}_{2} \mathrm{O}_{2}$ in wheat leaves after infestation by aphids. Control: untreated leaves; Sa48h: 48 h post S. avenae feeding; Sg48h: $48 \mathrm{~h}$ post S. graminum feeding. The values are presented as means \pm SE of three biological replicates. Different letters indicate significant differences among treatments $(P<0.05$, ANOVA) deposition induced by aphids is involved in the sealing of the sieve pores as a phloem defence mechanism that impedes mass flow and prevents the flow of nutrients to piercing-sucking insects [39].

Many studies have demonstrated that the damage symptoms induced by pathogens and herbivores in plants are caused by the secretion of plant cell wall enzymes during the process of pathogen infection and mirid Lygus hesperus feeding [40, 41]. Additionally, the activities of PEMs and PGs have also been detected in $S$. graminum watery saliva, and the injection of these commercial enzymes in plant leaves causes damage symptoms similar to those induced by $S$. graminum feeding $[42,43]$. However, pectinase activity has also been detected in the saliva of non-phytotoxic aphids, such as $S$. avenae and $A$. pisum $[43,44]$. Transmission electron microscopy has shown that stylets predominantly penetrate between the layers of cellulose fibres and not via the middle lamella pectin layer [45]. The role of the saliva pectinases of S. graminum in the induction of chlorosis remains unclear. Interestingly, in our study, many plant-derived enzymes and proteins involved in plant cell wall modifications, such as PGs, PEMs and expansins, were induced by $S$. graminum feeding, but none were induced by $S$. avenae feeding. The upregulation of PG and PEM activity might result in the degradation of the cell wall around aphid feeding sites. Plant cells exploit complicated mechanisms for sensing the loss of cell wall integrity (CWI) during biotic stress and activate a 


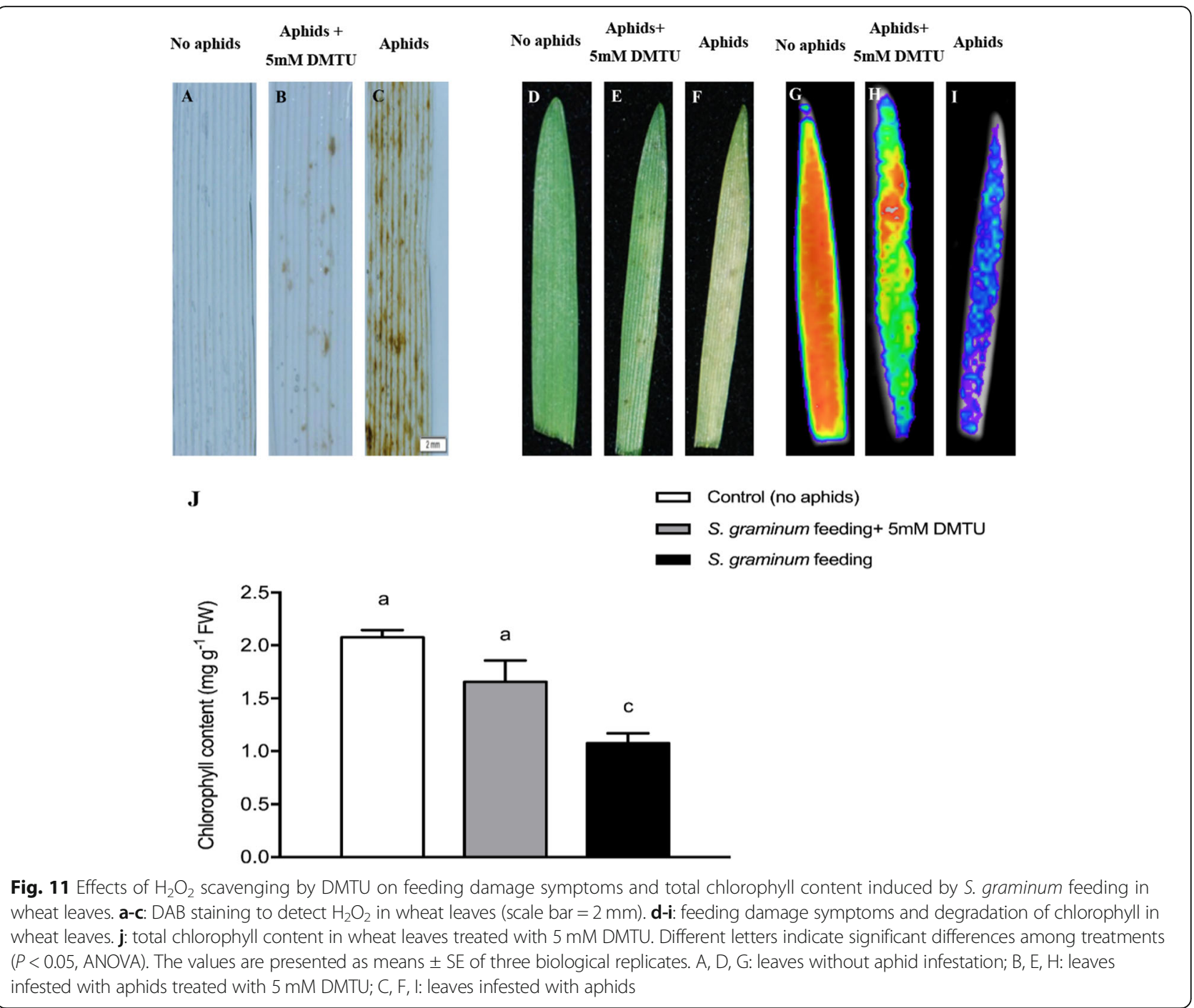

variety of defence responses [46]. For instance, the production of oligogalacturonic acid (OGA) fragments derived from the degradation of plant cell walls has been shown to trigger oxidative bursts, hypersensitive responses (HR), and other downstream defence responses in many plant species as a host-derived DAMP [47, 48], and these effects might further promote the induction of damage symptoms. In addition, ethylene production has been shown to be involved in the induction of plant cell wall-modifying proteins and the death of plant tissues. The role of PCMDPs and the ethylene pathway in the induction of chlorosis symptoms by $S$. graminum needs to be further investigated.

\section{S. graminum feeding induces strong ROS-scavenging activity in wheat leaves}

Aphid feeding usually leads to oxidative stress in host plants [49]. Oxidative stress is controlled by cellular antioxidant mechanisms in which multiple enzymatic scavengers, such as POD, APx, and CAT, are utilized by the cell to limit damage from reactive oxygen species $[50,51]$. The transcriptomic and enzymatic results showed that both $S$. graminum and $S$. avenae feeding increased the transcript levels and enzyme activities of ROS scavengers in wheat leaves. However, the expression levels and activity of antioxidants, particularly POD, induced by $S$. graminum feeding were notably higher than those induced by $S$. avenae, which suggested that $S$. graminum infestation results in strong oxidative stress and substantial $\mathrm{H}_{2} \mathrm{O}_{2}$ accumulation. Although ROS scavengers were significantly upregulated in response to $S$. graminum infestation, ROS production can exceed the cellular antioxidant capacity, resulting in oxidative damage to cellular components and cell death in leaves.

Induction of high $\mathrm{H}_{2} \mathrm{O}_{2}$ accumulation by $\mathrm{S}$. graminum feeding is involved in leaf chlorosis

$\mathrm{H}_{2} \mathrm{O}_{2}$ is involved in the activation of $\mathrm{HR}$, which is characterized by the rapid death of cells in the region 
surrounding the site of pathogen infection site [52, 53]. To further investigate the roles of $\mathrm{H}_{2} \mathrm{O}_{2}$ in the induction of feeding damage caused by $S$. graminum feeding, the accumulation of $\mathrm{H}_{2} \mathrm{O}_{2}$ in wheat leaves was detected. We found that $S$. graminum feeding induced the obvious accumulation of $\mathrm{H}_{2} \mathrm{O}_{2}$ at feeding sites, but $S$. avenae feeding had no significant effects on $\mathrm{H}_{2} \mathrm{O}_{2}$ production. In addition, seedlings treated with the $\mathrm{H}_{2} \mathrm{O}_{2}$ scavenger DMTU showed reductions in the chlorosis symptoms and chlorophyll loss triggered by $S$. graminum feeding. These results demonstrate that $\mathrm{H}_{2} \mathrm{O}_{2}$ accumulation plays important roles in the induction of chlorosis in wheat leaves in response to $S$. graminum feeding.

Aphid saliva is known to be involved in the induction of plant defence responses, and the eliciting activity of watery saliva of other aphid species such as $M$. persicae and $S$. avenae has been investigated $[54,55]$. The transient overexpression of Mp10, a salivary protein of M. persicae, induces plant defence responses and obvious chlorosis in N. benthamiana [56]. Specific elicitors or pathogen-like toxins in S. graminum saliva are likely involved in the induction of chlorosis. Comparative analyses of the salivary proteomes of four differentially virulent S. graminum biotypes revealed six salivary proteins with significant proteomic variation, and these proteins might thus be involved in the induction of feeding damage in plants [57]. Further research is required to identify the virulence factors in the salivary proteins of $S$. graminum and the mechanism underlying the induction of chlorosis.

\section{Conclusions}

In summary, the transcriptomic profiling of wheat performed in this study revealed similarities and differences among the responses of wheat to feeding by the phytotoxic aphid S. graminum and the non-phytotoxic aphid S. avenae. Both aphids induced the JA, SA and ET signalling pathways, but $S$. graminum triggered stronger plant defence responses and greater ROS-scavenging activity than $S$. avenae. A cytological analysis showed that aphid feeding induced callose deposition in wheat leaves and that substantial $\mathrm{H}_{2} \mathrm{O}_{2}$ accumulation was induced by S. graminum feeding. Our results also demonstrated that $\mathrm{H}_{2} \mathrm{O}_{2}$ plays vital roles in the induction of chlorosis in wheat leaves in responses to $S$. graminum feeding. Our future studies will focus on the mechanisms of $\mathrm{H}_{2} \mathrm{O}_{2}$ accumulation induced by $S$. graminum feeding and the roles of salivary proteins of $S$. graminum in the induction of chlorosis symptoms in wheat.

\section{Methods}

\section{Plants and aphids}

Seeds of Triticum aestivum var. Zhongmai 175 were germinated in distilled water for 3-4 days at a temperature of $25 \pm 1{ }^{\circ} \mathrm{C}$ in a Petri dish. Healthy seedlings of similar sizes were planted in $7.2 \times 7.2 \mathrm{~cm}$ plastic plots filled with organic soil and grown under controlled environmental conditions in climate chambers with a temperature of $20 \pm 1{ }^{\circ} \mathrm{C}$, a $40-60 \%$ relative humidity and a 14-h-light/ 10-h-dark photoperiod. Clones of $S$. graminum and $S$. avenae were maintained on the wheat plants (Zhongmai $175)$ as described previously [18].

\section{Aphid infestation}

At the two-leaf stage (12-day old plants), 20 apterous adult $S$. graminum or $S$. avenae were confined on the first leaf of wheat seedlings using a clip cage as described previously [18]. New-born nymphs produced by aphid adults were carefully removed every $12 \mathrm{~h}$ using a brush. After 48 $\mathrm{h}$ of feeding, all the aphids were removed, and leaf tissues of approximately $2.5 \times 2.5 \mathrm{~cm}$ from the aphid feeding sites of each plant were harvested flash frozen with liquid nitrogen and stored at $-80^{\circ} \mathrm{C}$ until further processing for RNA extraction. Detection of delayed fluorescence and histological staining were conducted immediately after sample collection. Three leaf sections covering the aphid feeding sites were collected from three independent plants and pooled to form one biological replicate. Three biological replicates were performed for each treatment.

\section{Changes in chlorophyll levels in wheat leaves after aphid infestation}

Delayed fluorescence is associated with extremely weak light emitted by chlorophyll molecules in plants and can reflect the chlorophyll content, providing a powerful tool for studying stress reactions in plants. The chlorophyll content in wheat leaves were each infested by 20 aphids as described above was first detected using the NightShade LB 985 In vivo Plant Imaging System (Berthold Technologies, Bad Wildbad, Germany). After $48 \mathrm{~h}$ of aphid feeding, the leaves were cut and immediately illuminated for $30 \mathrm{~s}$ with an LED panel. After the light was switched off, the delayed fluorescence was measured immediately using the NightShade system. The exposure time was set to $30 \mathrm{~s}$ using 4-by-4 pixel binning. The total chlorophyll content in wheat leaves after aphid infestation was also examined using a Chlorophyll Assay Kit (Solarbio, Beijing, China) according to the manufacturer's instructions. In brief, $0.1 \mathrm{~g}$ of fresh leaf tissues was ground to a fine powder and extracted with $2 \mathrm{~mL}$ of $80 \%$ acetone $(\mathrm{v} / \mathrm{v})$ at $4{ }^{\circ} \mathrm{C}$ for overnight. The homogenate was centrifuged at $4000 \mathrm{~g}$ for $10 \mathrm{~min}$ at $4{ }^{\circ} \mathrm{C}$, and the supernatant was used for the chlorophyll assay. The amounts of chlorophyll were detected spectrophotometrically, by reading the absorbance at 645 and $663 \mathrm{~nm}$ (DU800, Beckman, USA), and then calculated as described previously [58]. 
RNA preparation and sequencing library construction

Wheat leaves were first infested with aphids for $48 \mathrm{~h}$ as described above. The total RNA from the wheat leaves was extracted with the TRIzol reagent (Invitrogen) according to the manufacturer's recommended protocol. The RNA concentration was measured using a NanoDrop 2000 spectrophotometer (Thermo Fisher Scientific, Inc., USA), and the RNA integrity was evaluated using an Agilent 2100 Bioanalyzer (Agilent Technologies, Santa Clara, CA, USA). Samples with an RNA integrity number $(\mathrm{RIN}) \geq 7.0$ were used in the subsequent analysis. Libraries were constructed using the TruSeq Stranded mRNA LT Sample Prep Kit (Illumina, San Diego, CA, USA) according to the manufacturer's instructions and were sequenced on the Illumina sequencing platform (Illumina HiSeq 4000), which generated 150-bp paired-end reads were generated.

\section{RNA sequencing and data analysis}

The raw data (raw reads) were filtered to obtain highquality reads by removing the reads containing adaptor sequences, more than 3\% ambiguous bases (noted as $\mathrm{N}$ ) or more than 50\% low-quality bases (Phred quality score Q < $30)$. The resulting high-quality clean reads were then mapped to the reference genome (https://plants.ensembl. org/Triticum_aestivum/Info/Index) using TopHat2 with the default values [59]. The fragments per kilobase of exon model per million mapped reads (FPKM) data were used to estimate the transcript expression levels in all the samples, and the genes with more than 1 FPKM in at least one sample of wheat leaves were used for further analysis [60]. The differentially expressed genes (DEGs) between the control and treated samples were screened using DESeq based on the following criteria: adjusted $p$ value $(q$ value) threshold $<0.00001$ and $\left|\log _{2} \mathrm{FC}\right| \geq 1$. A PCA of nine different leaf samples was performed based on pairwise comparisons using the DESeq package of R [61]. GO enrichment and KEGG pathway enrichment analyses of the DEGs were performed using $\mathrm{R}$ based on the hypergeometric distribution [62]. To annotate the functions of the transcripts, the unigenes were blasted against the $\mathrm{Nr}$ database using the BLAST programme with an $E$-value $\leq 1 \mathrm{e}-5$.

\section{Assays of antioxidant enzymes in wheat leaves after aphid infestation}

The leaves were infested with 20 apterous adult S. graminum or $S$ avenae as described previously. The activities of peroxidase (POD), superoxide dismutase (SOD), catalase (CAT) and ascorbate peroxidase $(\mathrm{APx})$ in the wheat leaves at different time points after the aphid infestation (12 h, $24 \mathrm{~h}, 48 \mathrm{~h}$ and $72 \mathrm{~h}$ ) were examined using corresponding kits (Jiancheng Bioengineering Institute, Nanjing, China) according to the manufacturer's instructions. Briefly, $0.2 \mathrm{~g}$ of fresh leaf tissues was ground with
$1.5 \mathrm{~mL}$ of ice-cooled $50 \mathrm{mM}$ Na-phosphate buffer $(\mathrm{pH}=$ $7.8)$ containing $0.1 \mathrm{mM}$ EDTA and $1.0 \%(\mathrm{w} / \mathrm{v})$ polyvinylpyrrolidone. The homogenate was centrifuged at 15,000 $\mathrm{g}$ for $30 \mathrm{~min}$ at $4{ }^{\circ} \mathrm{C}$, and the supernatant was immediately collected for further enzyme assays. The activities of POD, SOD, CAT, and APx were measured by following the changes in absorbance at $470 \mathrm{~nm}, 560 \mathrm{~nm}, 240$ $\mathrm{nm}$ and $290 \mathrm{~nm}$, respectively, according to a previous study [63].

\section{Detection of $\mathrm{H}_{2} \mathrm{O}_{2}$ and callose accumulation in wheat leaves after aphid infestation}

The detection of $\mathrm{H}_{2} \mathrm{O}_{2}$ in wheat leaves by 3 '-diaminobenzidine (DAB) staining was performed according to the histochemical methods described by Wang et al. [64] with some modifications. In brief, leaf segments previously infested with 20 apterous adults of $S$. avenae or $S$. graminum were immersed in $1 \mathrm{mg} \mathrm{mL}^{-1} \mathrm{DAB}$ solution $\left(10 \mathrm{mmol} \mathrm{L}^{-1}\right.$ $\mathrm{Na}_{2} \mathrm{HPO}_{4}, \mathrm{pH} 3.8$ ), and incubated in the dark overnight at room temperature. Then, the leaves were decolorized in boiling $95 \%$ ethanol for $10 \mathrm{~min}$ and hyalinized in saturated chloral hydrate. The stained leaves were imaged using an Olympus BX-63 microscope (Olympus Corporation, Japan). The endogenous $\mathrm{H}_{2} \mathrm{O}_{2}$ content in the wheat leaves after aphid feeding was determined using the protocols reported by Ferguson et al. [65]. For the visualization of callose, the leaves were first fixated, destained overnight in 1:3 acetic acid/ethanol (v/v) solution and washed in $150 \mathrm{mM} \mathrm{K}_{2} \mathrm{HPO} 4$ for $30 \mathrm{~min}$. The leaves were subsequently incubated for $6 \mathrm{~h}$ with $150 \mathrm{mM} \mathrm{K}_{2} \mathrm{HPO} 4$ and $0.01 \%$ aniline blue for staining, and the callose depositions were observed and photographed with an Olympus SZX-16 fluorescence microscope (Olympus Corporation, Japan) using a DAPI filter.

\section{Wheat seedlings treated with DMTU solution}

The leaves of wheat seedlings were treated with $5 \mathrm{mM}$ dimethylthiourea (DMTU, a scavenger of $\mathrm{H}_{2} \mathrm{O}_{2}$ ) solution or deionized water (control) for $24 \mathrm{~h}$ and then infested with 20 apterous adults of S. graminum for $48 \mathrm{~h}$. Assessments of DAB staining and delayed fluorescence analyses and an assessment of the chlorophyll content of the wheat leaves were then performed as described previously.

\section{Statistics analysis}

All the data were analysed using SPSS Statistics 20.0 software (SPSS Inc., Chicago, IL., USA), and the differences between or among groups were examined through an independent-samples $t$-test or one-way analysis of variance (Duncan). $P$ values less than 0.05 were considered statistically significant. 


\section{Supplementary information}

Supplementary information accompanies this paper at https://doi.org/10. 1186/s12870-019-2148-5.

Additional file 1: Table S1. Summary of transcriptome data.

Additional file 2: Data S1. List of all DEGs after infestation by aphids in comparison with controls.

Additional file 3: Figure S1. KEGG enrichment.

Additional file 4: Data S2. DEGs involved in SA, JA and ET- dependent defence pathways in response to aphid feeding.

Additional file 5: Data S3. Transcript levels of genes involved in ROS scavenge after aphid feeding.

\section{Abbreviations}

ACO: 1-aminocyclopropane-1-carboxylate oxidase 1; ACS: 1aminocyclopropane-1-carboxylate synthase; AOC: Allene oxide cyclase; AOS: Allene oxide synthase; APx: Ascorbate peroxidase; BYDV: Barley yellow dwarf virus; CAT: Catalase; CWI: Cell wall integrity; DAB: 3'-diaminobenzidine; DAMPs: Damage associated molecular patterns; DEGs: Differentially expressed genes; DMTU: Dimethylthiourea; ET: Ethylene; EXP: Beta-expansin; FDR: Adjusted $p$ value; FPKM: Fragments per kilobase of exon model per million mapped reads; GPx: Glutathione peroxidase; $\mathrm{H}_{2} \mathrm{O}_{2}$ : Hydrogen peroxide; HAMPs: Herbivory associated molecular patterns; HR: Hypersensitive response; JA: Jasmonic acid; LOX: Lipoxygenase; MeJA: Methyl jasmonate; OGA: Oligogalacturonic acid; PAE: Pectin acetylesterase; PAL: Phenylalanine ammonia-lyase; PEM: Pectinesterase; PG: Polygalacturonase; PIs: Proteinase inhibitors; POD: Peroxidase; PR protein: Pathogenesis-related protein; RNA-Seq: High-throughput RNA sequencing; ROS: Reactive oxygen species; RuBisCO: Ribulose bisphosphate carboxylase oxygenase; SA: Salicylic acid; SEs: Sieve elements;

SOD: Superoxide dismutase

\section{Acknowledgements}

We would like to thank technician Ms. Yanxia Liu for aphid rearing in our laboratory.

\section{Authors' contributions}

$J L C$ and $Y Z$ designed the experiment, YZ, YF and JF performed the experiments, YZ, YF and QL analyzed the data, JLC and FF provided funding, YZ, JLC and FF wrote the first drafts of the manuscript. All authors critically read and approved the manuscript

\section{Funding}

This study was funded by the National Natural Science Foundation of China (31871979, 31901881), National Key R\&D Plan in China (2017YFD0201701, 2016YFD0300701), and the Cooperation Project between Belgium and China from MOST (2014DF32270). The funding organizations played no role in the design of study and collection, analysis, and interpretation of data and in writing the manuscript.

\section{Availability of data and materials}

The datasets used and analyzed during the current study are available from the corresponding author on reasonable request.

\section{Ethics approval and consent to participate}

The seeds of wheat, Zhongmai 175 were bred by institute of Crop Sciences, Chinese Academy of Agricultural and Sciences and bought from Henan Shengyuan Seed Industry Technology Co., Ltd., Xuchang, China. Cereal aphids Sitobion avenae and Schizaphis graminum used in our study were collected in Langfang, Hebei Province, China, and has been rear in our greenhouse for more than 9 years. The authors declare that all the experiments performed in this study comply with the institutional, national, or international guidelines.

\section{Consent for publication}

Not applicable.

\section{Competing interests}

The authors declare that they have no competing interests.

\section{Author details}

${ }^{1}$ State Key Laboratory for Biology of Plant Diseases and Insect Pests, Institute of Plant Protection, Chinese Academy of Agricultural Sciences, Beijing 100193, People's Republic of China. ${ }^{2}$ Functional and Evolutionary Entomology, Gembloux Agro-Bio Tech, University of Liège, B-5030 Gembloux, Belgium.

Received: 22 April 2019 Accepted: 19 November 2019

Published online: 10 December 2019

\section{References}

1. Howe GA, Jander G. Plant immunity to insect herbivores. Annu Rev Plant Biol. 2008;59:41-66.

2. Bonaventure G. Perception of insect feeding by plants. Plant Biol. 2012;14(6): 872-80.

3. Steppuhn A, Baldwin IT. Resistance management in a native plant: nicotine prevents herbivores from compensating for plant protease inhibitors. Ecol Lett. 2007;10(6):499-511.

4. Hartl M, Giri AP, Kaur H, Baldwin IT. Serine protease inhibitors specifically defend Solanum nigrum against generalist herbivores but do not influence plant growth and development. Plant Cell. 2010;22(12):4158-75.

5. Paré PW, Tumlinson JH. Plant volatiles as a defense against insect herbivores. Plant Physiol. 1999;121(2):325-32.

6. Kessler A, Baldwin IT. Defensive function of herbivore-induced plant volatile emissions in nature. Science. 2001;291(5511):2141-4.

7. Leitner M, Boland W, Mithöfer A. Direct and indirect defences induced by piercing-sucking and chewing herbivores in Medicago truncatula. New Phytol. 2005;167(2):597-606.

8. Heidel AJ, Baldwin IT. Microarray analysis of salicylic acid-and jasmonic acidsignalling in responses of Nicotiana attenuata to attack by insects from multiple feeding guilds. Plant Cell Environ. 2004;27(11):1362-73.

9. Mithöfer A, Wanner G, Boland W. Effects of feeding Spodoptera littoralis on lima bean leaves. II. Continuous mechanical wounding resembling insect feeding is sufficient to elicit herbivory-related volatile emission. Plant Physiol. 2005;137(3):1160-8.

10. Vos IA, Verhage A, Schuurink RC, Watt LG, Pieterse CMJ, van Wees SCM. Onset of herbivore-induced resistance in systemic tissue primed for jasmonate-dependent defenses is activated by abscisic acid. Front Plant Sci. 2013;4:539.

11. Kaloshian I, Walling LL. Hemipterans as plant pathogens. Annu Rev Phytopathol. 2005:43:491-521.

12. Coppola $\mathrm{V}$, et al. Transcriptomic and proteomic analysis of a compatible tomato-aphid interaction reveals a predominant salicylic acid-dependent plant response. BMC Genomics. 2013;14(1):515.

13. Donovan MP, Nabity PD, DeLucia EH. Salicylic acid-mediated reductions in yield in Nicotiana attenuata challenged by aphid herbivory. Arthropod Plant Interact. 2013;7(1):45-52.

14. Hu XS, Liu XF, Thieme T, Zhang GS, Liu TX, Zhao HY. Testing the fecundity advantage hypothesis with Sitobion avenae, Rhopalosiphum padi, and Schizaphis graminum (Hemiptera: Aphididae) feeding on ten wheat accessions. Sci Rep. 2015;5:18549.

15. Blackman RL, Eastop VF. Aphids on the world's crops: an identification and information guide. New York: John Wiley \& Sons Ltd.; 2000.

16. Miles PW. Aphid saliva. Biol Rev. 1999:74:41-85.

17. Al-Mousawi AH, Richardson PE, Burton RL. Ultrastructural studies of greenbug (Hemiptera: Aphididae) feeding damage to susceptible and resistant wheat cultivars. Ann Entomol Soc Am. 1983;76(6):964-71.

18. Zhang Y, Fan J, Fu Y, Francis F, Chen JL. Plant-mediated interactions between two cereal aphid species: promotion of aphid performance and attraction of more parasitoids by infestation of wheat with phytotoxic aphid Schizaphis graminum. J Agr Food Chem. 2019;67(10):2763-73.

19. Zhao LY, Chen JL, Cheng DF, Sun JR, Liu Y, Tian Z. Biochemical and molecular characterizations of Sitobion avenae-induced wheat defense responses. Crop Prot. 2009;28(5):435-42.

20. Zhu-Salzman K, Salzman RA, Ahn JE, Koiwa H. Transcriptional regulation of sorghum defense determinants against a phloem-feeding aphid. Plant Physiol. 2004;134(1):420-31.

21. Park SJ, Huang Y, Ayoubi P. Identification of expression profiles of sorghum genes in response to greenbug phloem-feeding using cDNA subtraction and microarray analysis. Planta. 2006;223(5):932-47. 
22. Sandström J, Telang A, Moran N. Nutritional enhancement of host plants by aphids-a comparison of three aphid species on grasses. J Insect Physiol. 2000;46(1):33-40.

23. Machado-Assefh CR, Lucatti AF, Alvarez AE. Induced senescence promotes the feeding activities and nymph development of Myzus persicae (Hemiptera: Aphididae) on potato plants. J Insect Sci. 2014;14:155.

24. Morkunas I, Mai VC, Gabryś B. Phytohormonal signaling in plant responses to aphid feeding. Acta Physiol Plant. 2011;33(6):2057-73.

25. Bi JL, Felton GW. Foliar oxidative stress and insect herbivory: primary compounds, secondary metabolites, and reactive oxygen species as components of induced resistance. J Chem Ecol. 1995;21(10):1511-30.

26. Blokhina O, Virolainen E, Fagerstedt KV. Antioxidants, oxidative damage and oxygen deprivation stress: a review. Ann Bot. 2002;91(2):174-94.

27. Choudhury S, Panda P, Sahoo L, Panda SK. Reactive oxygen species signaling in plants under abiotic stress. Plant Signal Behav. 2013;8(4):e23681.

28. Züst T, Agrawal AA. Mechanisms and evolution of plant resistance to aphids. Nat Plants. 2016;2(1):15206.

29. Wang XW, Li P, Liu SS. Whitefly interactions with plants. Curr Opin Insect Sci. 2017;19:70-5.

30. Reddy SK, Weng Y, Rudd JC, Akhunova A, Liu SY. Transcriptomics of induced defense responses to greenbug aphid feeding in near isogenic wheat lines. Plant Sci. 2013;212:26-36.

31. Moran PJ, Thompson GA. Molecular responses to aphid feeding in Arabidopsis in relation to plant defense pathways. Plant Physiol. 2001;125(2):1074-85.

32. Stewart SA, Hodge S, Bennett M, Mansfield JW, Powell G. Aphid induction of phytohormones in Medicago truncatula is dependent upon time postinfestation, aphid density and the genotypes of both plant and insect. Arthropod Plant Interact. 2016;10(1):41-53.

33. Hodge S, Bennett M, Mansfield JW, Powell G. Aphid-induction of defencerelated metabolites in Arabidopsis thaliana is dependent upon density, aphid species and duration of infestation. Arthropod Plant Interact. 2019; 13(3):387-99.

34. Argandoña VH, Chaman M, Cardemil L, Muñoz O, Zúñiga GE, Corcuera LJ. Ethylene production and peroxidase activity in aphid-infested barley. J Chem Ecol. 2001;27(1):53-68.

35. Malinovsky FG, Fangel JU, Willats WGT. The role of the cell wall in plant immunity. Front Plant Sci. 2014;5:178.

36. Voigt CA. Callose-mediated resistance to pathogenic intruders in plant defense-related papillae. Front Plant Sci. 2014;5:168.

37. Saheed SA, Cierlik I, Larsson KAE, Delp G, Bradley G, Jonsson LM, Botha CE. Stronger induction of callose deposition in barley by Russian wheat aphid than bird cherry-oat aphid is not associated with differences in callose synthase or $\beta-1$, 3-glucanase transcript abundance. Physiol Plantarum. 2009; 135(2):150-61.

38. Luna E, Pastor V, Robert J, Flors V, Mauch-Mani B, Ton J. Callose deposition: a multifaceted plant defense response. Mol Plant-Microbe Interact. 2011 24(2):183-93.

39. Will T, van Bel AJE. Physical and chemical interactions between aphids and plants. J Exp Bot. 2006;57(4):729-37

40. Kubicek CP, Starr TL, Glass NL. Plant cell wall-degrading enzymes and their secretion in plant-pathogenic fungi. Annu Rev Phytopathol. 2014;52:427-51.

41. de la Paz C-MM, et al. Polygalacturonase causes lygus-like damage on plants: cloning and identification of western tarnished plant bug (Lygus hesperus) polygalacturonases secreted during feeding. Arthropod Plant Interact. 2008;2(4):215.

42. Ma RZ, Reese JC, Black WC IV, Bramel-Cox P. Chlorophyll loss in a greenbugsusceptible sorghum due to pectinases and pectin fragments. J Kansas Entomol Soc. 1998;71(1):51-60.

43. Cherqui A, Tjallingii WF. Salivary proteins of aphids, a pilot study on identification, separation and immunolocalisation. J Insect Physiol. 2000; 46(8):1177-86.

44. Liu Y, Wang WL, Guo GX, Ji XL. Volatile emission in wheat and parasitism by Aphidius avenae after exogenous application of salivary enzymes of Sitobion avenae. Entomol Exp Appl. 2009;130(3):215-21.

45. Tjallingii WF, Esch TH. Fine structure of aphid stylet routes in plant tissues in correlation with EPG signals. Physiol Entomol. 1993;18(3):317-28.

46. Hématy $\mathrm{K}$, Cherk $\mathrm{C}$, Somerville $\mathrm{S}$. Host-pathogen warfare at the plant cell wall. Curr Opin Plant Biol. 2009;12(4):406-13.

47. Ferrari S, Savatin DV, Sicilia F, Gramegna G, Cervone F, De Lorenzo G. Oligogalacturonides: plant damage-associated molecular patterns and regulators of growth and development. Front Plant Sci. 2013;4:49.
48. Lee $\mathrm{S}$, et al. Oligogalacturonic acid and chitosan reduce stomatal aperture by inducing the evolution of reactive oxygen species from guard cells of tomato and Commelina communis. Plant Physiol. 1999;121(1):147-52.

49. Bednarski W, Borowiak-Sobkowiak B, Wilkaniec B, Samardakiewicz S, Morkunas I. Oxidative stress in pea seedling leaves in response to Acyrthosiphon pisum infestation. Phytochemistry. 2013;93:49-62.

50. Mittler R. Oxidative stress, antioxidants and stress tolerance. Trends Plant Sci. 2002;7(9):405-10.

51. Demidchik V. Mechanisms of oxidative stress in plants: from classical chemistry to cell biology. Environ Exp Bot. 2015;109:212-28.

52. Thordal-Christensen $\mathrm{H}$, Zhang Z, Wei Y, Collinge DB. Subcellular localization of $\mathrm{H}_{2} \mathrm{O}_{2}$ in plants. $\mathrm{H}_{2} \mathrm{O}_{2}$ accumulation in papillae and hypersensitive response during the barley-powdery mildew interaction. Plant J. 1997;11(6): 1187-94.

53. Govrin EM, Levine A. The hypersensitive response facilitates plant infection by the necrotrophic pathogen Botrytis cinerea. Curr Biol. 2000;10(13):751-7.

54. De Vos M, Jander G. Myzus persicae (green peach aphid) salivary components induce defence responses in Arabidopsis thaliana. Plant Cell Environ. 2009;32(11):1548-60.

55. Zhang Y, Fan J, Francis F, Chen JL. Watery saliva secreted by the grain aphid Sitobion avenae stimulates aphid resistance in wheat. J Agr Food Chem. 2017:65(40):8798-805.

56. Bos JIB, Prince D, Pitino M, Maffei ME, Win J, Hogenhout SA. A functional genomics approach identifies candidate effectors from the aphid species Myzus persicae (green peach aphid). PLoS Genet. 2010;6(11):e1001216.

57. Nicholson SJ, Puterka GJ. Variation in the salivary proteomes of differentially virulent greenbug (Schizaphis graminum Rondani) biotypes. J Proteome. 2014;105:186-203.

58. Arnon DI. Copper enzymes in isolated chloroplasts: polyphenoloxidase in Beta vulgaris. Plant Physiol. 1949;24(1):1-15.

59. Kim D, Pertea G, Trapnell C, Pimentel H, Kelley R, Salzberg SL. TopHat2: accurate alignment of transcriptomes in the presence of insertions, deletion and gene fusions. Genome Biol. 2013;14(4):36.

60. Mortazavi A, Williams BA, McCue K, Schaeffer L, Wold B. Mapping and quantifying mammalian transcriptomes by RNA-Seq. Nat Methods. 2008:5:621-8.

61. Anders S, Huber W. Differential expression of RNA-Seq data at the gene level-the DESeq package. Heidelberg: European Molecular Biology Laboratory (EMBL); 2012.

62. Kanehisa $M$, et al. KEGG for linking genomes to life and the environment. Nucleic Acids Res. 2008;36:480-4.

63. Jiang $L$, Yang $H$. Prometryne-induced oxidative stress and impact on antioxidant enzymes in wheat. Ecotoxicol Environ Saf. 2009;72(6):1687-93.

64. Wang CF, Huang LL, Buchenauer H, Han QM, Zhang HC, Kang ZS. Histochemical studies on the accumulation of reactive oxygen species $\mathrm{O}_{2}{ }^{-}$ and $\mathrm{H}_{2} \mathrm{O}_{2}$ ) in the incompatible and compatible interaction of wheat Puccinia striiformis f. sp. tritici. Physiol Mol Plant Pathol. 2007;71(4-6):230-9.

65. Ferguson IB, Watkins CB, Harman JE. Inhibition by calcium of senescence of detached cucumber cotyledons effect on ethylene and hydroperoxide production. Plant Physiol. 1983;71(1):182-6.

\section{Publisher's Note}

Springer Nature remains neutral with regard to jurisdictional claims in published maps and institutional affiliations.

Ready to submit your research? Choose BMC and benefit from:

- fast, convenient online submission

- thorough peer review by experienced researchers in your field

- rapid publication on acceptance

- support for research data, including large and complex data types

- gold Open Access which fosters wider collaboration and increased citations

- maximum visibility for your research: over $100 \mathrm{M}$ website views per year

At BMC, research is always in progress.

Learn more biomedcentral.com/submissions 